\title{
MedFilm. Enseigner " par et pour la recherche » les SHS en milieu médical à l'aide du film « utilitaire »
}

\author{
Christian Bonah ${ }^{\mathrm{a}}$ et Joël Danet ${ }^{\mathrm{b}}$ \\ SAGE, UMR 7363, Université de Strasbourg, France
}

\begin{abstract}
Résumé. MedFilm est un projet enseignement-recherche par et sur le film « utilitaire » médical et sanitaire. Le film "utilitaire » peut être défini ici sommairement comme produit pour une finalité spécifique et pragmatique différente du divertissement ou de l'expression artistique. Les enseignements proposés basés sur des sources audiovisuelles utilisées comme ressources pédagogiques entendent mobiliser ces dernières comme des « outils pédagogiques transitionnels », à savoir des documents référents, jouant un rôle de tiers, qui permettent d'ouvrir la séquence pédagogique à une réflexion partagée. Pour élargir le regard des étudiants et les sensibiliser aux enjeux des sciences et des techniques dans nos sociétés contemporaines, ces ressources témoignent de politiques de santé, évoquent les représentations de la nature d'une maladie, les modalités de prévention et de soins qu'il convient d'analyser à travers un détour par le film utilitaire historique. En réponse aux besoins des enseignements, le projet de recherche MedFilm, concernant le repérage et l'analyse systématique des films utilitaires médicaux et sanitaires français entre 1895 et 1960, a élaboré la plate-forme numérique d'enseignement et de recherche MedFilm. Aaccessible en ligne, elle héberge à la fois un corpus de films documentaires sanitaires et médicaux, leur analyse sous forme de fiches individuelles, ainsi que des documents annexes afin de les rendre accessibles pour la formation et la recherche dans le domaine des SHS en milieu scientifique.
\end{abstract}

\begin{abstract}
MedFilm is a research and teaching project with and about medical and health related utility film. Utility film may be defined for our purpose as film produced for reasons other than entertainment or as art work. It pursues specific and pragmatic sponsor's goals. Teaching with audio-visuals uses these resources as transitional tools for instruction. They become reference documents, used as independent propositions, opening a teaching session that aims at collaborative analysis and mutually elaborated understanding. To widen student's horizon and point of view and to foster their receptiveness to challenges of better understanding science and technology in our contemporary societies, these audiovisuals portray health policies and politics, evoke representations of nature and disease, present practices of prevention and cure that can be analyzed through the detour with historical health related utility film. Working towards teaching with film, the research project MedFilm is concerned with the identification and the systematic analysis of medical and health related utility films in France between 1895 and 1960. The project has produced
\end{abstract}

\footnotetext{
a e-mail : bonah@unistra.fr

b e-mail : danet@unistra.fr
}

This is an Open Access article distributed under the terms of the Creative Commons Attribution License 4.0, which permits unrestricted use, distribution, and reproduction in any medium, provided the original work is properly cited. 


\section{SHS Web of Conferences}

an online digital film database useful for research and teaching. It comprises a digital corpus of health and medical films and their analysis presented in form of media-Wiki pages describing the films. Furthermore it gathers archival sources concerning the films in order to make these available for research and teaching in medical humanities and beyond.

\section{Introduction}

Le projet MedFilm présenté ici se situe dans un contexte pédagogique déterminé par des facteurs d'horizons différents. D'une part, l'intérêt que présente aujourd'hui le film médical et sanitaire, d'abord comme source historiographique, puis en tant qu'outil pédagogique pour enseigner les SHS en médecine, pharmacie et en sciences de la vie. D'autre part la transformation de l'enseignement de l'histoire des sciences et des techniques, particulièrement en milieu médical et scientifique. Parti d'une réflexion et d'une pratique d'enseignement de SHS en médecine, le projet a évolué vers la construction progressive d'un projet de recherche à proprement parler. Celui-ci a été soutenu dans le cadre d'un programme de recherche MedFilm financé par l'Agence Nationale de la Recherche (ANR) pour la période 2010-2014. ${ }^{1}$ Son extension du projet de recherche a mené à la construction de la plate-forme pédagogique et de recherche MedFilm en collaboration avec la Direction des Usages du Numérique de l'Université de Strasbourg. Cette plate-forme, accessible en ligne, est dédiée à l'enseignement des sciences humaines en études de santé et en sciences de la vie. ${ }^{2}$

MedFilm constitue un outil de recherche pour stimuler une historiographie des sciences de la vie et de la santé à partir de, et sur les films médicaux et sanitaires, devenus source et objet de recherche. MedFilm situe dans le même temps ces films dans le cadre d'enseignements. Dès le départ, la conception de la plate-forme envisage d'associer à ses contenus les travaux d'étudiants et de chercheurs confirmés. Elle est ainsi conçue à l'image des démarches du milieu du $19^{\mathrm{e}}$ siècle à l'origine des disciplines et universités scientifiques modernes ${ }^{3}$ Il s'agit d'une initiative qui associe étroitement enseignement et recherche dans des échanges bidirectionnels permanents. La plate-forme répond aux besoins d'un « impératif de recherche ${ }^{4}$ tout en favorisant un enseignement « pour et par la recherche $»^{5}$ associant les étudiants directement à celle-ci.

La suite de cette contribution revient sur la genèse du projet selon les trois points évoqués plus haut : l'intérêt que présentent les films comme sources de recherche et supports pédagogiques, les transformations de l'enseignement des SHS en médecine, enfin les premières expériences pédagogiques

\footnotetext{
${ }^{1}$ MedFilm, Instruire, informer, communiquer, éduquer. Le film médico-sanitaire en France, 1895-1960, Programme BLANC 2010, ANR-10-BLAN-2004.

2 http://medfilm.unistra.fr. Tous les liens internet ont été consultés en date du 06/05/2014.

${ }^{3}$ Nous désignons comme « université moderne » les institutions qui après la réorganisation suivant les principes néo-humanistes au début du $19^{\mathrm{e}}$ siècle, s'appuient sur trois principes fondamentaux : l'unité de la science, l'unité de l'enseignement et de la recherche, et l'idéal de «Bildung » (éducation). Schubring, 1991, pp. 276-326, en particulier p. 278.

La mise en place d'un « disciplinary order of sciences » entre 1780 et 1840 forme le point central des études de R. Stichweh sur la "genèse du système scientifique moderne » organisé sous forme de disciplines scientifiques marques par leurs «impératifs de recherche » Stichweh, 1991. Turner, 1986, pp. 495-502.

4 "Science is method not information. ...Science teaching is properly teaching by collaboration, not by affirmation." Flexner, 1912, pp. 119-122. Il ajoute quelques pages plus loin: "As to research: be a man never so busy with patients, teaching, or what not, no member of a German clinic can do his full duty by faithful attention to routine. Research is in the air", Idem, p. 166.

5 Billroth soulignait cette transformation de l'enseignement médical au XIXème siècle qui s'était faite en deux phases distinctes : la première du commentaire du livre vers la démonstration par le professeur, la seconde de la démonstration professorale vers l'investigation par les étudiants. « Une des conséquences de la revendication de la liberté de l'enseignement concerne le fait que l'enseignant doit initier l'étudiant à l'esprit et à la méthode de la recherche scientifique » Billroth, 1876, pp. 36- 42. « On entend de la part des professeurs de manière générale qu'il n'est pas nécessaire de traiter dans les cours toutes les matières de manière exhaustive, mais qu'il suffirait de stimuler l'élève pour que celui-ci se sentît motivé à étudier par lui-même et pour lui-même. ». Idem., p. 61. Voir également autour de la notion de «Lehrfreiheit» et du principe de l'unité de l'enseignement et de la recherche : Paulsen, 1921.
} 
engagées. Dans un deuxième temps nous approfondirons notre réflexion sur la façon dont l'implication des films en tant qu'outils pédagogiques a accompagné l'évolution des cours. Pour finir, nous reviendrons sur la création de la plate-forme MedFilm, son utilisation et sa valorisation : d'une part dans une extension des enseignements dans le cadre des cours de première années commune d'études de santé (PACES), d'autre part dans des enseignements multiples de SHS en médecine, en pharmacie et en sciences de la vie, intégrant les travaux d'étudiants à la constitution même de la plate-forme. Notre travail de recherche a pour objet d'étudier les films médico-sanitaires français à partir d'ensembles cohérents, comme des dispositifs de communication spécifiques en recherche, enseignement, éducation sanitaire et promotion industrielle. Il est nécessaire d'adosser chacun de ces dispositifs à un environnement social, culturel, historique et technique. Il est aussi nécessaire de les analyser en tant qu'agencements d'images animées et de sons. C'est de cette façon que nous les abordons comme producteurs de représentations médico-sanitaires spécifiques. Nous expliquerons en quoi ces films nous paraissent constituer des « outils pédagogiques transitionnels », à savoir des documents référents, jouant un rôle de tiers, qui permettent d'ouvrir la séquence pédagogique à une réflexion partagée.

\section{Genèse d'un projet enseignement-recherche ${ }^{6}$ par et sur le film « utilitaire » médical et sanitaire}

Notre constat initial part de l'observation : «Que serait la vie sans mémoire ? Que serait la mémoire sans images ? Que serait l'image de notre temps sans le film ?» ${ }^{7}$. Les images contribuent à façonner notre compréhension du monde ${ }^{8}$. L'invention du cinéma est un fait essentiel dans l'histoire des rapports entre sciences, médecine et société au XXe siècle. Les films constituent en outre des supports fascinants pour observer la diffusion massive des représentations médicales et scientifiques de la « réalité », et leur influence sur les notions que leur public se fait de la santé et la maladie. Pour l'historien les « moving pictures $\gg$ du $20^{\mathrm{e}}$ siècle tiennent lieu de source et d'observatoire. Concernant le film médical et sanitaire, différents types de production sont en jeu. Il peut s'agir de films réalisés par des médecins qui se positionnent également comme cinéastes ou des collaborations entre scientifiques et réalisateurs stricto sensu; de films produits par des compagnies cinématographiques, et destinés à des publics constitués de médecins et de scientifiques ou au grand public. Commandités par des entreprises ou des institutions en rapport avec la santé et la médecine, ils intègrent des programmes de formation professionnelle ou des campagnes sanitaires. A notre époque de disponibilité digitale, ce patrimoine audiovisuel est aujourd'hui marqué par un paradoxe. Jusque dans les années 1980-1990, les travaux historiques sur ces films ont rencontré des difficultés liées à leur confidentialité. Dans les années récentes, ils sont devenus de plus en plus accessibles à travers des mises en ligne sous l'égide d'initiatives différentes. Nous pouvons citer les archives Prelinger ${ }^{9}$ aux Etats-Unis, depuis 2002 intégrées à la Library of Congress Motion Picture, Broadcasting and Recorded Sound Division. Nous pouvons aussi citer la National Library of Medicine (NLM) avec son « Historical Audiovisuals program ${ }^{10}$, ou la Wellcome Library à Londres

\footnotetext{
${ }^{6}$ La désignation « enseignement-recherche » avec un trait d'union signifie ici qu'il ne s'agit pas seulement d'un projet avec un volet recherche et un volet enseignement, mais que le projet, comme la suite du texte le montrera, est caractérisé par des aller-venues incessantes entre des questions de recherche et d'enseignement consubstantielles de son élaboration et de son fonctionnement. En ce sens le trait d'union entre recherche et enseignement renvoie au caractère imbriqué et intégré des deux activités dans le cadre du projet MedFilm.

${ }^{7}$ Maxime des Archives du film de la Friedrich-Wilhelm-Murnau-Stiftung : http://www.murnau-stiftung.de consulté en date du 06/05/2014.

8 Belting, 2004.

${ }^{9}$ https: //archive.org consulté en date du 06/05/2014.

10 http://www.nlm.nih.gov/hmd/collections/films consulté en date du 06/05/2014.
} 
avec la création de la « Moving Image and Sound Collection $» .{ }^{11}$ En France, le Centre de ressources et d'informations sur les multimédias pour l'enseignement supérieur (CERIMES) a mis en ligne une collection de films historiques, en lien avec la vidéothèque de l'enseignement supérieur. ${ }^{12}$ Toutefois, sans un minimum de contextualisation, ces sources restent souvent inintelligibles et donc peu exploitées. Il devient donc nécessaire de les situer, à la fois pour la recherche et l'enseignement.

Depuis vingt ans, l'enseignement des sciences humaines et sociales (SHS) en médecine, comme dans le milieu scientifique dans son ensemble, tend à se transformer en France ${ }^{13}$, comme plus largement en Europe et en Amérique du Nord. ${ }^{14}$ L'un de ses enjeux consiste à susciter des approches nouvelles pour rendre accessibles aux étudiants des contenus qui accompagnent leur formation scientifique et technique. Ceux-ci leur proposent une " mise en culture » des savoirs et des savoir-faire qu'ils acquièrent dans leurs formations principales. ${ }^{15}$ Pour élargir le regard des étudiants et les sensibiliser aux pratiques du passé et aux enjeux présents des sciences et des techniques dans nos sociétés contemporaines, l'une des approches défendues consiste à recourir à des documents audiovisuels historiques et contemporains. ${ }^{16}$

Cette évolution de fond recoupe la démarche entreprise depuis quinze ans par une équipe d'enseignants-chercheurs au sein du Département d'Histoire des Sciences de la Vie et de la Santé (DHVS) à la faculté de médecine de Strasbourg. Les enseignements de SHS qu'elle a conçus, lesquels s'appuient sur des sources audiovisuelles, ont d'abord concerné un module optionnel, "Cinéma, littérature et médecine », proposé en $2^{\mathrm{e}}$ et $3^{\mathrm{e}}$ année des études médicales. L'expérience pédagogique s'est généralisée et transformée progressivement. Le choix initial a été d'employer des films de fiction de distribution commerciale, à l'image de la base de données « Literature, Arts \& Medicine Database » de la New York University School of Medicine. ${ }^{17}$ Cependant, ce choix bien que légitime et utile en soi, présente des limites pédagogiques. Les descriptions fictionnelles grand public peuvent se révéler éloignées de la « réalité » vécue par les étudiants en parcours professionnel. Dans de tels films, les sciences et la médecine servent souvent de vecteurs narratifs plutôt que de véritable thématique. La mise en scène des épisodes historiques et des thématiques scientifiques et médicales traitées y présente souvent un caractère anecdotique voire anachronique. Ainsi pour élargir et approfondir l'approche initiale, notre intérêt s'est davantage porté vers les films de non-fiction. Films documentaires d'auteur d'abord, films « utilitaires » ensuite. ${ }^{18}$ Le film « utilitaire » peut être défini ici

\footnotetext{
11 http://wellcomelibrary.org/about-us/about-the-collections/moving-image-and-sound-collection consulté en date du 06/05/2014. Ce site propose une description assez détaillée des films mis en ligne.

12 http: //www. canal-u.tv consulté en date du 06/05/2014.

13 Voir, pour la réforme du 18 mars 1992 qui introduit l'enseignement des SHS en médecine en France, les textes précis des différents arrêtés voir les annexes du supplément de la Chronique mensuelle que l'AUFEMO a publiées le 1er avril 1996. Ce supplément de 100 pages, préfacé par le père de la réforme de 1992, le conseiller Jean Rey, dresse la situation des SHS en médecine en 1996. Les textes des arrêtés se trouvent également sur le site du journal officiel de République française : www. légifrance.gouv.fr consulté en date du 06/05/2014. Pour un regard retrospectif : Bonah, 2006, pp. 9-18. Neuman, 2005, pp. 9-10.

14 Pour l'enseignement de l'histoire des sciences et des techniques en général voir à titre d'exemple : Debru, 1999. Belhoste, Bruno, Gispert, Hélène, Hulin, Nicole (éds.), 1996. Fauque, 2006. http://trema.revues .org/83, mis en ligne le 01 octobre 2008, consulté le 06 mai 2014. A titre d'exemple et pour une perspective internationale de l'enseignement de l'histoire des sciences et des techniques en médecine/santé : Bonah, Rasmussen (éds.), 2005.

15 Pour une synthèse : Bonah, Haxaire, Mouillie, Penchaud, Visier (éds), 2011. Bonah, Rasmussen (éds.), 2005.

16 Self, 1993, pp. 383-385. Boisaubin, Winkler, 2000, pp. 292-296. Blasco, 2001, pp. 426-428. Lepicard, Fridman, 2003, pp. $1039-1040$.

${ }_{17}$ Une des premières initiatives en ce sens est la « Literature, Arts \& Medicine Database » de la New York University School of Medicine. Elle se définit comme une liste annotée de ressources multimédia de littérature, poésie, film, vidéo et art, développée pour constituer une ressource dynamique, accessible exhaustive pour l'enseignement et la recherche en « medical humanities ». La base ne contient pas les films décrits mais seulement leur description et analyse, puisque ce sont des films de fiction commerciales, donc couverts par des droits d'exploitation et accessibles par la vente. En revanche la base établit des liens très intéressants entre les trois domaines de la littérature, le film et les arts plastiques et leurs rapports avec la santé et la médecine. http://litmed.med.nyu. edu consulté en date du 06/05/2014.

18 Pour la définition et la terminologie du « film utilitaire Gebrauchsfilm ». Elsaesser, 2009, pp. 19-34.
} 
sommairement comme produit pour une finalité spécifique et pragmatique différente du divertissement ou de l'expression artistique. Dits également films « inédits et éphémères », ils partagent la mission de transmettre un message. ${ }^{19}$ Tel film, qui témoigne de la condition des malades de la lèpre sur l'île de Spinalonga (L'ordre, 1974, de Jean-Daniel Pollet), est une production à l'initiative des Laboratoires Pharmaceutiques Sandoz ${ }^{20}$; Tel autre film, qui détaille les méfaits d'un fléau et vante les mérites du dépistage précoce (Le Cancer, fléau social, 1927, de Jean-Benoit-Lévy) est commandité par l'Institut du Cancer de la Faculté de Médecine de Paris avec le concours du Ministère de l'Hygiène mais aussi de la Ligue des Sociétés de la Croix-Rouge. ${ }^{21}$, Tel film télévisuel de treize minutes, où trois scientifiques sont interviewés séparément pour répondre aux interrogations des jeunes sur le dépistage du Sida, le rôle du virus sur le système immunitaire et sur la prévention contre le sida par le préservatif dans les années 1980 (Virus, quel virus ?, 1987, de Fabrice Rouleau) a été commandé par l'association de malades AIDES et la Caisse nationale de l'assurance maladie des travailleurs salariés . ${ }^{22}$ D'un point de vue informatif, ces documents sont souvent dépassés quelques années après leur production. Ils sont alors retirés de la circulation. Difficilement accessible par la suite, ils sont pourtant riches en informations concernant les représentations qu'une société se fait à un moment donné d'une maladie, de la santé, de la relation entre professionnels scientifiques, médecins et publics.

Éclectiques et faites des hasards de rencontres, les premières recherches de films permettent à l'un des auteurs de ce texte, dans le cadre d'une démarche d'abord individuelle, de tisser progressivement un réseau de collaborations : avec de rares chercheurs travaillant dès les années 1990 sur ce sujet ${ }^{23}$, avec un employé temporaire d'une coopérative culturelle de cinéma rural- qui n'est d'autre que le co-auteur de ce texte ${ }^{24}$, avec des enseignant-chercheurs dans des UFR de cinéma, arts du spectacle

\footnotetext{
${ }^{19}$ Les qualificatifs « inédits et éphémères » recoupent pour une partie la catégorie des films « utilitaires». Produits comme ces dernières pour une finalité spécifique et pragmatique différente du divertissement ou de l'expression artistique ils partagent souvent la mission de transmettre un message. La durée limitée de la validité ou de la pertinence du message rend ces films, au bout d'un certain temps, obsolètes par rapport à leur utilité initiale. Par exemple, un film de prévention sanitaire de 1987 au sujet du Sida perd son utilité lorsque son contenu devient inexact dans le cadre des avancés de la recherche ; un film de promotion industriel n'est plus utile lorsque l'installation ou les produits mis en valeur ne sont plus d'actualité. En ceci, ces films sont éphémères puisque leur projection et sauvegarde deviennent très aléatoire une fois leur message périmé. La qualification de films «inédits » renvoie à l'idée que ces films souvent coproduits par un commanditaire et une entreprise de production de films n'entrent pas dans un circuit de distribution commercial. Contrairement aux films de grande distribution, leur absence de catalogues de distribution commerciale fait disparaitre ces films souvent lors de restructurations des institutions ou des entreprises commanditaires. Non revendiqués, ces films peuvent devenir alors des films « orphelins », c'est-à-dire des films à statut indéterminé quant à leur ayantsdroit. Le qualificatif «inédits » inclut également des films amateurs pour lesquels une diffusion publique n'est pas envisagée initialement.

${ }^{20}$ Le film longtemps « inédit » et « éphémère » a été rendu accessible en 2006 par la sortie du DVD : Jean-Daniel Pollet - Tours d'horizon - 5 films : Méditerranée, Bassae, L'ordre, Dieu sait quoi, Ceux d'en face, Pom Films, 2006. De ce fait il ne se trouve pas sur la plate-forme MedFilm. Pour Jean-Daniel Pollet voir sa notice sur Ciné-Ressources, le catalogue collectif des bibliothèques et archives de cinéma : http://cinema. encyclopedie.personnalites.bifi.fr/index.php?pk=11584 consulté en date du 06/05/2014. Cet exemple montre le caractère fluctuant et imparfait des catégories explicitées à la note précédente.

21 http://medfilm.unistra.fr/wiki/Le_cancer_fl\%C3\%A9au_social__ $281927 \% 29$ consulté en date du 06/05/2014.

22 http://medfilm.unistra.fr/wiki/Virus,_quel_virus_\%3F_\%281987\%29 consulté en date du 06/05/2014.

23 Pharmacien, historien et docteur en études cinématographiques et audiovisuelles de formation initiale, Thierry Lefebvre a soutenu en 1996 une thèse intitulée Cinéma et discours hygiéniste (1890-1930). Depuis 1997 maitre de conférences en sciences de l'information et de la communication, il enseigne à l'Université Paris 7 Didérot et a publié un nombre considérable de textes sur le cinéma médical. Thierry Lefebvre n'est pas seulement intervenu dans des séances de cours au fil des années, mais il a aussi partagé un certain nombre de copies de films qui furent parmi les tout premiers films utilitaires utilisés en cours. Qu'il soit remercié à cet endroit pour ses connaissances partagées sur le sujet et pour son ouverture d'esprit et de collaboration. http://thierry-lefebvre.blogspot.fr/; http://lefebvre-th.monsite-orange.fr/ et http://sciences-medias.fr/blogs/equipe/thierry-lefebvre/ consultés en date du 06/05/2014.

${ }^{24}$ Historien de formation initiale, Joël Danet a travaillé depuis les années 1990s dans le domaine de l'éducation à l'image pour la Coopérative Régionale Cinéma Culturel (C.R.C.C.) et l'association Vidéo-les-beau-jours (http://www. videolesbeauxjours.org/ consulté en date du 06/05/2014). Depuis le début des années 2000 il participe à la conception et la tenue de cours qui s'appuient sur l'utilisation du film et représente le plus ancien collaborateur des projets présentés ici. Il est aujourd'hui chercheur contractuel à l'Université de Strasbourg, et chargé de cours dans les UFRs de médecine et d'arts du spectacle-cinéma, responsable de nombreuses initiatives de formation à l'image pour le Rectorat de Strasbourg
} 


\section{SHS Web of Conferences}

et d'information et de communication ${ }^{25}$, et enfin des responsables de cinémathèques et d'archives audiovisuelles. Dans ce processus, la quête de films de «non-fiction » en tant que supports pédagogiques rencontre des difficultés multiples. Premièrement, et de façon générale, les lieux les plus évidents pour accéder à ces sources audiovisuelles, les cinémathèques et les archives du film, ne fournissent pas un inventaire accessible au public de leurs films. Autrement dit il faut d'abord connaitre le titre d'un film (pourtant souvent imprécis ou pas connu du chercheur) afin de demander si l'institution en question en possède une copie. Si des demandes thématiques sont possibles en principe, leur résultat est très variable et la question d'un traitement suffisant, adéquat voire exhaustif des sources, importante pour l'historien, reste dans ce domaine à la discrétion entière de l'institution interrogée. ${ }^{26}$ Deuxièmement, l'identification et la localisation d'un film une fois aboutie, son visionnage se fait sur place. S'il permet l'analyse d'une œuvre individuelle et de sa pertinence - ou non - pour un enseignement, se pose ensuite la question de la possibilité d'obtenir une copie de travail ou d'enseignement et les frais inhérents à cette mise à disposition. Les contraintes techniques et financières couvrent ici un éventail qui va de la numérisation d'un film pellicule $(35,16 \mathrm{~mm}$ ou autres formats) à la recherche des ayant droits souvent difficiles à identifier. Dans le meilleur des cas les archives ou cinémathèques disposent d'une copie vidéo du document, dont la multiplicité des formats (VHS, BETA, etc.) et la rareté des appareils lecteurs, limitent désormais l'exploitation. S'il existe des copies numériques, elles ne concernent de loin pas l'intégralité de la production visée. Ces questions de difficulté d'accès aux sources audiovisuelles utilitaires, éphémères ou inédites, bien connues des chercheurs évoluant dans le domaine de l'histoire du film documentaire ${ }^{27}$, sont d'autant plus importantes lors qu'il s'agit de les mobiliser pour des enseignements. Là où un visionnage sur place et sur table de montage peut suffire pour la recherche, l'utilisation pédagogique d'un film nécessite sa mise à disposition ou sa copie afin de pouvoir le projeter en cours ou de le revoir à plusieurs reprises pour une étude détaillée. ${ }^{28}$ Ces difficultés d'accès expliquent en partie le fait que les études sociales des sciences et l'histoire des sciences hésitent encore à appréhender le film comme source historique à part entière.

L'obtention de copies de films individuels a permis progressivement de multiplier le recours à ces matériaux dans le cadre de divers enseignements. L'accueil favorable de ces « objets pédagogiques ${ }^{29}$ par les étudiants à conduit à son tour à la création de nouveaux enseignements Ils ont donné lieu à

ainsi que responsable de cycles de projections rencontres (par exemple « Filmer la ville ») pour le pôle régional d'éducation artistique et de formation au cinéma et à l'audiovisuel (Vidéolesbeauxjours), dont le siège est la Maison de l'Image de Strasbourg. Depuis les années 2000, nous co-organisons également des projections rencontres régulières et publiques sur le thème «santé et société » à la Maison de l'Image de Strasbourg en collaboration avec Vidéolesbeauxjours et l'INA. La dernière manifestation de ce type était : "Histoire(s) de télévision : Jour après jour - nos vies devant la caméra. La pudeur ou l'impudeur de Hervé Guibert », lundi 17 février 2014. http://www.videolesbeauxjours. org/index.php?option=com_rencontre\&view=rencontre\&Itemid=27\&idCycle=8\&idArticle=558 consulté en date du 06/05/2014.

25 Aujourd'hui professeur en Sciences de l'information et de la communication à l'Université de Lorraine, Vincent Lowy est spécialiste de l'histoire et de la théorie du cinéma classique et du film documentaire. Il dirige depuis juillet 2013 l'Institut Européen de Cinéma et d'Audiovisuel (ICEA : http://www.univ-lorraine.fr/content/instituteurop $\%$ C3\%A9en-de-cin\%C3\%A9ma-et-daudiovisuel-ieca consulté en date du 06/05/2014) à Nancy et est membre du Centre de recherche sur les médiations (CREM, EA 3476). http://crem.univ-lorraine.fr/ consulté en date du 06/05/2014. Au moment du début de notre collaboration Vincent Lowy était maître de conférences en Arts du spectacle-Cinéma à l'Université de Strasbourg. Pour ses publications voir la bibliographie. http://crem.univ-lorraine.fr/chercheurs/Lowy.html consulté en date du 06/05/2014.

${ }^{26}$ Ce constat initial et pragmatique est le point de départ sur lequel a été fondé le projet de recherche présenté dans la deuxième partie de cette contribution.

27 Voir à titre d'exemple : Leblanc, 1983. Pessis, 1997. Zimmermann, 2011.

28 Pour répondre à cette difficulté, le projet MedFilm fait le choix sur sa plate-forme pédagogique et de recherche de rendre accessible les films et leur analyse pour rendre possible leur projection en cours ou leur étude détaillée sous forme d'un accès individuel des étudiants via l'environnement numérique de travail (ENT) de leur institution d'enseignement supérieur d'appartenance. Activer l'onglet « Login via Single Sign-on » en haut à droite des pages de la plate-forme.

29 Voir pour une élaboration théorique de leur utilisation la partie suivante de la contribution. 
des modules optionnels thématiques avec des intitulés comme : "Portraits de patients », "Mediator - médiateurs- médicamenteurs : Médicaments et scandale », "Souffrance, médecine et guerre », «L'autre comme "monstre » : entre mise en scène et mise en abîme », ou encore "L'hôpital et ses représentations : soins, vie, travail ». A partir de l'année 2005 un ensemble de 75 h de cours assurées par 3 enseignants chercheurs du DHVS s'appuient sur ces films comme support pédagogique associé à des documents littéraires, artistiques et historiques. Le DHVS a constitué progressivement une vidéothèque sous forme de copies VHS et DVD pour une utilisation sous forme d'extraits ou en visionnage intégral. Cet usage s'est diffusé vers d'autres ensembles d'enseignements, notamment des cours d'histoire des sciences en milieu scientifique ${ }^{30}$ et dans le cadre du Master « Sciences, technologies et société : Histoire, philosophie et médiation des sciences » à l'Université de Strasbourg. ${ }^{31}$ Au fil des enquêtes menées pour localiser et contextualiser les films, trois évolutions se sont fait jour. (1) L'utilisation des films dans un cadre pédagogique appelait une réflexion plus approfondie sur leur statut, leur utilité et leur raison d'être en tant que ressource pédagogique. (2) L'étendue des recherches engagées à leur sujet suggérait de construire un projet de recherche sur l'histoire du film médical et sanitaire en France. (3) Les premières communications et publications au sujet des films médicaux, ainsi que les cours, amenait, de la part d'autres enseignants-chercheurs, la demande régulière de pouvoir disposer de certains films. Or cette demande restait difficile à honorer, compte tenu des restrictions faites par les archives détentrices des documents de ne pas communiquer les copies de travail qu'elles avaient mis à disposition à des chercheurs individuels. Après cette description narrative de la genèse du projet MedFilm, la suite de cette contribution revient plus en détail sur les problématiques et les résultats de recherche de chacune des trois étapes esquissées.

\section{Les sources audiovisuelles comme ressources pédagogiques}

«Qu'est-ce que vous avez fait en cours ? - Nous avons regardé un film. - Ah.... A Au prime abord les ressources audiovisuelles sont souvent perçues comme le palliatif ou substitut d'un contenu de cours. Les a priori vont parfois jusqu'au soupçon. Au milieu des années 2000, la commande d'un DVD «Looney Tunes. Tes héros préférés », une compilation de grands classiques complètement déjantés du cinéma d'animation pour enfants de la Warner Bros. Entertainment Inc. nous a valu une enquête administrative demandant de justifier cette dépense d'une valeur d'une dizaine d'euros. Il a fallu répondre que le bonus du DVD contient un document important : le film d'animation à but préventifSo much for so little (Etats-Unis, 1949) ${ }^{32}$ illustrant la vie du petit John E. Jones. Le film montre que l'enfant risque, s'il ne reçoit pas des soins de première enfance appropriés, de rejoindre les 118.481 nouveau-nés par an (sur 2 millions) qui décèdent pendant leur première année de vie aux Etats Unis dans l'après Deuxième Guerre mondiale. A la fin du film, le message est clair : 3 cents par Américain et par semaine permettraient de pourvoir aux soins nécessaires pour sauver plus de 100.000 enfants comme John annuellement, justifiant le titre du film « Autant pour si peu».

Au-delà des stéréotypes et des réserves concernant l'utilisation du film comme ressource pédagogique (en dehors des études universitaires de cinéma, évidemment), y recourir impliquait pour nous une réflexion sur le bien-fondé, les enjeux et les difficultés d'une telle démarche. ${ }^{33}$ Les raisons pour

\footnotetext{
${ }^{30}$ Par exemple : « Histoire et épistémologie des neurosciences », UE du Master de Neurosciences ; « Histoire de l'innovation thérapeutique », UE du Master Sciences du médicament en pharmacie.

31 http://mastersts.u-strasbg.fr consulté en date du 06/05/2014.

32 Le film est dirigé par Charles M. Jones et produit par Edward Selzer pour le compte du Public Health Service de la Federal Security Agency des Etats-Unis.Looney Tunes. Tes héros préférés. Volume 3, Warner Kids, DVD restauré, remasteurisé en version intégrale, Warener Bros. Entertainment Inc, 2004. So much for so little est le $16^{\mathrm{e}}$ film sur ce DVD qui se trouve dans le bonus.

33 Pour cette approche dans le domaine francophone voir : Rasmussen, 2005, pp. 90-93. Lepicard, 2005, pp. 85-87. Bonah, 2005, pp. $81-84$.
} 
lesquelles nous utilisons des films aujourd'hui restent multiples, à la fois d'ordre pratique et d'ordre théorique. ${ }^{34}$

En pratique, un film montré aux étudiants devient une référence commune avec le professeur qui l'a choisi. Il suscite une immersion rapide et collective dans une situation présentielle, et permet souvent de proposer une mise en situation concrète et réelle, mais conditionnelle : après tout, il s'agit du point de vue d'un réalisateur-auteur, que celui-ci l'assume ou non. Pour la construction d'un contenu de cours à partir du film, l'avantage est que celui-ci fournit une référence tierce, proposée par l'enseignant, mais autonome. A mi-chemin entre l'enseignant et les enseignés elle est plus facilement discutable et critiquable que la parole de l'enseignant qui fait autorité. ${ }^{35}$ En ce sens nous caractérisons les films, en tant que ressources pédagogiques, comme des « objets pédagogiques transitionnels ». Les films constituent dans ce cadre un support préparant une réflexion autonome sur l'entrée à l'hôpital, la relation médecin-malade, l'examen clinique, etc. Au-delà de la fonction que l'enseignant lui a attribuée, le film possède ses caractéristiques propres. La projection d'un film, depuis l'origine du cinéma, invite tout spectateur à une projection de soi. Il cherche à provoquer et à mobiliser une implication synonyme d'une ouverture émotionnelle. Ceci est vrai pour les films de fiction et pour les films utilitaires historiques avec l'avantage pour ces derniers de cibler particulièrement des questions concernant des pratiques professionnelles. Des interrogations sur un passé partagé sont ainsi renvoyées au spectateur d'aujourd'hui. Le détour par des films d'un passé divergent peut interroger les pratiques et identités actuelles. Par une interaction avec les étudiants, ces films très situés visent à exploiter cette expérience faite d'un mélange d'étrangeté et d'identité. Se déplacer à travers ces films « utilitaires », mobilisés comme ressources pédagogiques, implique de prendre en compte la spécificité de leur économie. Plus que les fictions, les films utilitaires sont associés à un contexte de production locale et enracinés dans leur espace de communication. Parmi les objectifs de leur utilisation dans le cadre d'un cours, il est nécessaire de les étudier comme moyens utilitaires produits par et/ou projetés devant des publics médico-scientifiques professionnels ou dans des espaces publics plus généraux. Il s'agit alors de les approcher en tant que phénomène culturel pour lui-même et comme observatoire historique du $20^{\mathrm{e}}$ siècle sur les pratiques et les représentations autour de la santé et des sciences de la vie et de la santé. Puisque même le cinéma documentaire conserve une part subjective, partielle et construite, le détour par le film cherche à créer une prise de recul ouvrant un espace réflexif. Sous un angle plus théorique, le film et l'œuvre d'art de manière plus générale engagent un regard qui crée des dispositifs alternatifs pour analyser et penser la réalité. Le film, en l'occurrence, acquiert une fonction de miroir. ${ }^{36}$ C'est une référence tierce qui implique le spectateur à ce titre. Son regard extérieur permet d'élaborer l'analyse des situations qu'il met en scène à la fois selon une approche par problématique, c'est-à-dire par les thématiques abordées et pour lesquelles le film est choisi - qu'il témoigne de politiques de santé, évoque les représentations de la nature d'une maladie, les modalités de prévention et de soins - et selon un ancrage disciplinaire SHS.

Un exemple concernant les enseignements théoriques monitorés du Troisième cycle de médecine générale permet d'illustrer notre propos. Depuis 2010, une formation commune médecine générale - SHS en médecine d'une durée de $12 \mathrm{~h}$ et intitulé «Devenir médecin - être médecin » cherche à inciter les étudiants jeunes internes à prendre un temps à part dans leur pratique quotidienne pour lire et analyser des documents à même de les faire réfléchir, en-deçà des aléas de leur vie professionnelle,

\footnotetext{
${ }^{34}$ Les élaborations qui suivent s'appuient sur notre expérience avec le film utilitaire. Toutefois, ces affirmations s'appliquent pour une part tant au film de non-fiction qu'au film de fiction. Par ailleurs, la distinction entre fiction et non-fiction pour nombre de documents est imparfaite en ce sens que le film utilitaire, de commande et à message associe souvent des éléments documentaires et fictionnels.

$35 \mathrm{Si}$ cette observation peut s'appliquer également à un texte, le film possède l'avantage d'une mise en commun rapide, dense et partagée sur place. La lecture, si elle se fait sur place aurait un même effet. Une lecture à distance de l'enseignement pose essentiellement le problème de l'adhérence des enseignés.

36 Lepicard, 2005, pp. 85-87. Bonah, 2005, pp. 81-84.
} 
sur leurs motivations pour devenir ou être médecin. Par un regard croisé médecine générale - SHS en rupture avec les formes d'enseignement classiques de transmission de connaissances, le cours cherche à analyser avec les internes des situations concrètes dans l'optique d'une co-construction des savoirs par les enseignants et les enseignés. Si le principe est de travailler à partir de situations vécues, la première séance de formation se déroule néanmoins avec la projection d'un film. L'introduction de la référence tierce permet de prolonger les récits des étudiants, d'aller au-delà du partage d'expériences avec les autres, de trouver des références à mêmes de penser ces situations vécues, de prolonger la réflexion par un apport théorique. ${ }^{37}$ La perception par les étudiants de ce dispositif est exprimée par l'un d'eux comme suit :
«Pour en venir à un point plus précis, le film, en tant qu'introduction a évité une entrée en matière trop brutale par une mise à nu immédiate de nos difficultés. Il apportait une représentation extérieure à la médecine, celle d'un cinéaste, plus confortablement critiquable dans un premier temps qu'une situation que nous avions vécue. En effet une situation réelle est autrement plus difficile à aborder car elle implique de dévoiler une part de ses faiblesses et de ses limites, et nécessite parfois de déstabiliser la hiérarchie très ancrée dans la formation médicale.
Le travail personnel a été pour moi la partie la plus riche en enseignements, car il permet de prendre du temps et du recul par rapport au cours. Le cours m'a permis d'aborder mon exercice médical en construction avec un nouveau regard, plus critique et interrogateur qu'auparavant. J'ai été convaincu que les situations sources de difficulté étaient tout autant source de progrès et d'enseignement. Cette façon d'enseigner m'a donné, plus encore que le goût de la connaissance, le goût de la remise en question et de la découverte personnelle. Le travail écrit a donné un support idéal à cette réflexion. » ${ }^{38}$

La validation des enseignements proposés repose sur le même principe, qu'il s'agisse des cours de « cinéma, littérature et médecine » thématiques, ou bien d'enseignements spécifiques qui ne font recours au film que de manière transitionnelle comme dans le cas évoqué ci-dessus. Elle requiert généralement un travail personnel des étudiants sur des sujets choisis et validés par les enseignants. Il en a résulté la production de dizaines de mémoires originaux et souvent de qualité par les étudiants. Certains se sont prolongés sous forme de mémoires de master voir de thèses de médecine. C'est une source de grande satisfaction pour les enseignants et les étudiants, c'est aussi le regret de voir ces travaux s'accumuler sans autre valorisation que leur fonction docimologique. Ainsi est née l'idée de leur trouver, par le biais de MedFilm, une forme de publicisation et d'accessibilité autre que leur dépôt dans une archive morte ou au mieux semi-vivante. ${ }^{39}$ Réalisée en 2013, la plate-forme pédagogique MedFilm permet en effet d'associer des étudiants en tant que rédacteurs de fiches (associés aux films mis en ligne) ${ }^{40}$ et de documents annexes aux fiches et aux films. Ainsi, certains travaux d'étudiants intègrent depuis cette date la plate-forme. Rendus visibles, ils contribuent à contextualiser les films. A titre d'exemple, le travail de Master de Caroline Ruebrecht au sujet des films médico-psychiatriques d'Eric Duvivier constitue

${ }^{37}$ Les ressources utilisées dans ce cadre incluent à titre d'exemple des films de fiction comme : A tombeau ouvert de Martin Scorsese (Etats-Unis, 1999) ou Stormy weather de Soveig Anspach (France-Islande-Belgique, 2003). L'inconvénient de ces films de fiction pour l'enseignement est leur durée relativement longue de 2 heures en moyenne. Les films utilitaires utilisés incluent $L a$ journée d'un médecin de Nestor Almendros (France, CNDP, 1967) Le médecin de Philippe Haudiquet et André Teisseire (France, CNDP, 1969) disponibles sur la plate-forme MedFilm et d'une durée moyenne de 30 minutes ou encore des films documentaires comme Les patients de Claire Simon (France, 1989) ou La consultation de Hélène de Crécy (France, 2007) qui ont une durée moyenne de 90 minutes.

38 Communication « Devenir médecin - être médecin. Relecture croisée enseignants-enseignés d'une expérience pédagogique expérimentale collaborative entre médecine générale et SHS » présenté dans le cadre du Congrès des sciences humaines en médecine et santé « discours, récits en santé » à Amiens les 20,21, 22 juin 2013. Le texte reproduit ici correspond à la réflexion de Thomas Blanck, étudiant en premier semestre du Troisième cycle des études médicales (TCEM) et interne en médecine générale à la faculté de médecine de Strasbourg.

${ }^{39}$ En tant que copie d'examen au Département d'Histoire des Sciences de la Vie et de la Santé de la faculté de médecine de Strasbourg.

40 Voir l'onglet administrateur des fiches de la plate-forme MedFilm. 
la base des fiches correspondantes de la plate-forme MedFilm. Son mémoire y figure en outre comme document associé téléchargeable. ${ }^{41}$ De manière analogue, le travail de thèse en médecine de Maryse Contal concernant l'analyse de 29 films de prévention entre 1987 et 1999 contre le Sida en France l'a amené à rédiger les fiches correspondant à son corpus. Des documents y sont joints en annexe, incluant une frise chronologique des films rappelant leur contexte socio-politique, ainsi que le manuscrit de thèse à proprement parler. ${ }^{42}$

En 2009-2010 le temps était venu de construire un projet de recherche concernant le repérage et l'analyse systématique des films utilitaires français produits pour des campagnes de santé, les films éducatifs et d'information dans le cadre scolaire et professionnel, les films industriels et de recherche et même les films réalisés sans finalité sanitaire prioritaire, qui témoignent néanmoins de manière indirecte d'un regard sur ce domaine. Le corpus de films visés a en commun leur caractère éphémère par la validité limitée des messages dont les films sont porteurs. Difficilement accessible, ces documents sont pourtant riches en informations concernant les représentations qu'une société se fait à un moment donné d'une maladie, de la santé, de la relation entre professionnels scientifiques, médecins et publics. La maladie considérée comme fait social, fléau ou stigmate, la relation patient-soignant, les enjeux idéologiques que recouvrent les politiques sanitaires successives, motivent différentes formes de discours : communication officielle de l'institution médicale, information polémique de la presse, intimité créative de l'individu artiste, questionnement réflexif du philosophe : le projet de recherche MedFilm envisage d'interroger de manière historique et audiovisuelle les formes et les mutations de la communication dans le domaine de la santé à partir d'une analyse systématique du corpus des premiers films cinématographiques utilitaires et sanitaires entre 1895 et 1960.

\section{De l'enseignement à la recherche : le programme de recherche MedFilm}

Plus de 400000 films utilitaires ont été produits et dorment comme documents éphémères dans des centres d'archives, dépôts, caves, ou disparaissent tout simplement. ${ }^{43}$ Grâce à la révolution numérique, qui est aussi une révolution de l'audiovisuel, ce type de films devient disponible. Pour leur sauvegarde et leur valorisation, et l'un implique bien l'autre, un premier objectif principal du projet de recherche MedFilm est d'identifier ces documents de manière systématique et de constituer un corpus cohérent de films pour les décrire et les analyser. Le besoin de disposer de copies de travail de ces films pour leur analyse détaillée et approfondie rentre en résonnance avec notre souci de fournir un accès libre et pratique à ces ressources à une large population d'étudiants et d'enseignants francophones. Leur accessibilité est aussi nécessaire dans le cadre d'un enseignement qui met à profit une telle production d'analyses et connaissance.

En établissant un état de l'art pour le projet, nous considérions que des rapports étroits existent entre le développement de la recherche scientifique et médicale et celui du film documentaire, voire du film de divertissement de masses. Il est toutefois surprenant de constater que les récentes études d'histoire culturelle ou d'histoire du film documentaire demeurent timides quant au « film de faits »

\footnotetext{
41 Ruebrecht, 2012, 175 pages. http://medfilm.unistra.fr/wiki/Documents_attachés_à_la_fiche consulté en date du 06/05/2014. Un autre mémoire de Master est actuellement en préparation sous la direction d'Alexandre Sumpf sur les films produits depuis les années 1930 par les Potasses d'Alsace.

42 Contal, 2013. Soutenance le 17 Septembre 2013. http://medfilm.unistra.fr/wiki/Documents_attachés_à_la_ fiche_ consulté en date du 06/05/2014.

43 Pour le chiffre de 400000 films qui concerne le monde occidental pour la période entre 1898 et 2000 voir : Vonderau, 2009, pp. 51-61, ici p. 53. Une estimation approximative considère qu'un tiers de ces films n'existe plus aujourd'hui. La sauvegarde de ce patrimoine devrait être une préoccupation majeure pour les institutions concernées et pour la société plus globalement.
} 
médico-sanitaire ${ }^{44}$ - qu'il s'agisse de films de recherche et d'enseignement médicaux ou de films de prévention. Si les histoires du documentaire, en Allemagne de Jung et Loiperdinger ${ }^{45}$, en Grande Bretagne de Boon ${ }^{46}$, excellentes au demeurant, évoquent régulièrement le film scientifique et médical, elles persistent pour beaucoup à le considérer comme une production anecdotique, d'ordre purement technique et pédagogique. En France, une histoire synthétique du film documentaire faisait et fait encore généralement défaut. De même, si l'ambitieuse entreprise que représentent les trois volumes de $l^{\prime}$ ' « Histoire du corps ${ }^{47}$ » porte une attention soutenue aux représentations du corps, qu'il soit normal ou anormal, dans la culture de masse, et notamment dans les fictions cinématographiques, elle ne dit rien des liens que ces œuvres tissent avec les documentaires médico-sanitaires qui leurs sont contemporains.

Dans le domaine de l'histoire, des études audiovisuelles et de l'histoire des médias et de la communication en France, se saisir du film en tant que source date des années 1970. Aujourd'hui cette démarche engage de nouveaux questionnements ${ }^{48}$. De source complémentaire et anecdotique, le film est devenu une source à part entière munie de son propre statut épistémologique. ${ }^{49}$ L'histoire des sciences et des médias, cependant, n'a pas assez pris la mesure ni du rôle que jouent les « films de faits » médicosanitaires pour l'ère antérieure à l'apparition de la télévision, ni du potentiel que détiennent ces contenus audio-visuels entre 1895 et 1960 en tant qu'objets propres et comme modalité de " mise en société » des sciences et des techniques. Parmi les travaux menés sur le traitement médiatique des sciences et de la médecine ${ }^{50}$, peu ont été consacrés à l'étude des rapports entre sciences, médecine et société dans les films utilitaires de la première moitié du 20e siècle. ${ }^{51}$ En France, les études sociales des sciences et l'histoire des sciences hésitent encore à appréhender le film comme source historique à part entière. Pourtant, dans nombre de travaux anglo-saxons et allemands, le film scientifique et médical a pris une importance grandissante ces vingt dernières années, que ce soit en histoire sociale et culturelle ou en histoire des sciences biomédicales. ${ }^{52}$ A partir de leurs contenus, les auteurs se saisissent de thèmes aussi divers que l'expérimentation humaine ${ }^{53}$, la sexualité et la reproduction ${ }^{54}$ ou les biographies de personnalités scientifiques ${ }^{55}$. Mais la plupart des analyses sont fondées sur la lecture d'un unique film, sans toujours justifier de son choix particulier. Si des travaux plus ambitieux existent pour certains groupes sociaux ${ }^{56}$ ou certaines maladies ${ }^{57}$, une approche systématique sous la forme d'une analyse d'un corpus cohérent de films fait encore largement défaut. Même à échelle internationale, peu de recherches s'interrogent sur les rapports entre les films de recherche, d'information et d'éducation sanitaire et le reste de la production filmique, documentaire ou de fiction. Une telle omission laisse supposer que les

\footnotetext{
44 Initialement le projet de recherche désignait le corpus de films visés comme « films de faits médico-sanitaires » en empruntant ce terme aux travaux de Timothy Boon (films of facts). Boon, 2008. Il nous a paru progressivement que cette désignation essentialisait les « faits » en question et que le terme de film « utilitaire » semblait plus adéquat, signifiant et surtout moins connoté par rapport à la question de l'objectivité des « faits » scientifiques et médicaux. Elsaesser, 2009, pp. 19-34. Zimmermann, 2009, pp. 101-117. Zimmermann, 2011.

45 Jung, Loiperdinger, 2005. Kreimeier, Ehmann, Goergen, 2005. Zimmermann, Hoffmann, 2005.

46 Boon, 2008. Boon, 1999.

47 Corbin, Courtine, Vigarello (éds.), 2006.

48 Ferro, 1977/1993. Osolin, 1988. Delage, 1989. Gerverau, 2000. Guibbert, Oms, 1993. Fleury, 1998.

49 Delage, Guigueo, 2004.

50 Chervin, 2003. Cheveigné, Chervin, Sorlin, 1977.

51 A l'exception des travaux de T. Lefebvre : Lefebvre, 1996. Lefebvre, 2004. Pastre, Lefebvre, 2012. Martinet, 1994. Vignaux, 2007.

52 Ostherr, 2005. Ostherr, 2004, pp. 299-314. Schmidt, 2002, pp. 23-46. Boon, 1999. Parascandola, 1996, pp. 173-175. Pernick, 1996. Pernick, 1978, pp. 21-27. Cantor, 2007, p. 39-69. 2005. Orgeron, Orgeron, Streible, 2011.

53 Krause, Pethes, 2007. Lederer, 2007, pp. 94-115.

54 Kuhn, 1988. Keitz, 2005.

55 Reim, 1990, pp. 22-33.

56 Köhne, 2009. Podoll, Lüning, 1998, pp. 122-132.

57 Riehl-Halen, 1998. Cantor, 2007, 81, pp. 39-69. Ellenbrand, 1999.
} 
films utilitaires médico-sanitaires ne répondraient pas à une intention d'écriture, ne feraient que refléter une réalité objective. Leur étude détaillée démontre le contraire.

Cet état de l'art conduit à deux conclusions majeures : Tout d'abord sur un plan international, les historiens et les historiens des sciences prennent de plus en plus en compte le statut des images, animées ou non, comme vecteur essentiel de l'éducation populaire, de l'éducation à la santé et de la rhétorique scientifique. En France, ce travail reste à développer, particulièrement dans le domaine des films utilitaires médico-sanitaires. Autre constatation : si nous disposons actuellement d'études de cas sur certains sujets scientifiques ou sur des films individuels, il manque une approche systématique des films de faits médico-scientifiques en tant que dispositif de médiation et en tant que modalité d'éducation sanitaire.

Le deuxième objectif principal du projet de recherche MedFilm vise ainsi à combler, du moins partiellement, ces lacunes par l'étude systématique des sources filmiques utilitaires françaises, considérées comme vecteurs éducatifs et dispositifs de médiation entre sciences, médecine et société. Ces films constituent des supports fascinants pour observer la diffusion massive des représentations médicales et scientifiques de la "réalité », et leur influence sur les notions que leur public se fait de la santé et la maladie. Dans le domaine scientifique, l'image prétend produire des preuves et suggère l'enregistrement d'une « réalité». Ainsi l'image participe à établir des « faits » scientifiques. En même temps, il faut rappeler que dès l'origine du cinéma, l'image est employée pour présenter et simplifier des phénomènes scientifiques complexes. Elle montre, démontre et explique. En ce sens l'image est à la fois une forme de communication et une forme de médiation entre sciences, médecine et sphère publique. C'est pour mettre en perspective le déploiement du savoir scientifique en société que le projet envisage d'interroger de manière historique et audiovisuelle les films de faits médico-sanitaires français entre 1895 et 1960. La problématique de recherche esquissée convient au statut donné à ces documents dans le cadre des enseignements décrits auparavant : des dispositifs de médiation basés sur leur réactivation « en différé », dont la finalité pédagogique et éducative est d'inviter à une prise de distance par la relecture d'un passé en rapport avec le présent. D'une certaine manière il s'agit d'historiciser le factuel ou de penser un fait scientifique ou médical comme un moment où un évènement dans l'histoire de la pensée et de sa mise en pratique. Le projet de recherche rejoint ici l'une des problématiques mêmes de l'enseignement des SHS dans un milieu professionnel expert.

Il parait enfin utile d'enrichir les travaux sur la médiation entre sciences et société et sur l'éducation sanitaire par des travaux sur la circulation d'images et leurs dispositifs de projection. Ceux-ci, dans le cadre préventif ou de formation, tendent à créer leurs propres codes et contexte de référence. Une troisième ligne d'argumentation du projet concerne la ré-actualisation des campagnes de prévention et le renforcement des politiques d'éducation à la santé depuis le début des années $2000 .{ }^{58}$ Face à des menaces sanitaires nouvelles et d'ampleur mondiale, les Etats occidentaux et les organismes internationaux de santé réinvestissent l'idée de la prévention dans le cadre de la nouvelle santé publique. Depuis le début du $20^{\mathrm{e}}$ siècle, « savoir prévenir » revient à recourir à des techniques de communication audiovisuelle «modernes ». Afin de mettre en perspective la communication sanitaire actuelle, le programme de recherche MedFilm envisage d'interroger de manière historique les formes et les mutations de la communication par le film dans le domaine de la prévention sanitaire. Une analyse des modalités de production des premiers films d'information et de prévention médico-scientifiques jusqu'en 1960, ainsi que des compétences impliquées dans leur réalisation et leurs diffusions et usages, est nécessaire pour préciser leur contexte. L'enjeu est de mieux comprendre la circulation d'images scientifiques comme dispositifs de médiation des savoirs et des représentations biomédicales jusqu'à aujourd'hui. Étudier les films utilitaires médico-scientifiques conduit par ailleurs à s'interroger sur les mises en scène des pouvoirs et des savoirs scientifiques et sur la possibilité de distinguer, au sein

58 Bonah, Visier, 2011, pp. 268-275. 
de leur discours, la part de «propagande » au service des politiques de santé. Une telle démarche met en perspective et analyse les techniques audio-visuelles utilisées jusqu'à aujourd'hui dans les campagnes de prévention et d'éducation à la santé. Elle entre en résonance avec l'objectif pédagogique des enseignements présentés dans la partie précédente, à savoir la prise de distance critique sur l'actualité au moyen d'un détour par le film utilitaire historique.

A partir de ce cadrage initial, le projet de recherche MedFilm repère, localise, décrit, analyse et rend accessible de manière systématique un corpus de plus de 350 films utilitaires médicaux et sanitaires qui sont aujourd'hui méconnus et inaccessibles. Il répond par-là à l'absence d'inventaire systématique des films utilitaires à thématique de santé en France. Les auteurs de la Geschichte des dokumentarischen Films in Deutschland expliquent le point aveugle que constituent les films utilitaires médico-sanitaire par cette hypothèse : «Le film de recherche scientifique et le film de prévention n'étaient pas évoqués dans la presse spécialisée sur le cinéma, que ce soit par des annonces ou des critiques. Une approche pertinente du film de recherche scientifique et médical, et du film d'information et de prévention sur la santé, requerrait de se plonger dans la littérature professionnelle de ces disciplines, produite tout au long de leur histoire. » Le projet MedFilm réalise les premières étapes d'un tel inventaire. Il consiste ensuite à décrire et à analyser un corpus homogène, circonscrit et maîtrisable de films par une étude systématique. La connaissance intime de ce corpus initie dès lors une étude détaillée du film utilitaire médico-sanitaire français, selon trois questionnements : (1) comment des images produites dans un contexte scientifique identifié présentent et représentent les savoirs concernés ; (2) comment les images qui sont produites pour - et diffusées dans - l'espace public orientent et façonnent la perception commune des sciences, technologies et de la médecine ; (3) comment mettre en évidence les dispositifs communicationnels qui véhiculent ces images, l'impact du medium audiovisuel, la portée de l'art cinématographique au service du message, mais aussi les contraintes que la fabrication du film, les enjeux de réalisation, de production et de diffusion imposent à la mise en œuvre du discours d'information et de prévention.

Le projet de recherche MedFilm est ainsi conçu comme un observatoire des documents audiovisuels dans le domaine de la santé de ses origines filmiques jusqu'à l'arrivée de la télévision dans les années 1950-1960. La méthodologie du projet repose sur deux démarches : la comparaison d'une part, les «visual studies »d'autre part. Empruntée aux travaux engagés par l'histoire comparée et l'histoire croisée, l'approche comparative ${ }^{59}$ a été adaptée pour l'analyse des films. L'objet de cette méthode est de dépasser la singularité de chacun des documents filmiques pour les intégrer dans leur contexte sociopolitique en France et dans le contexte cinématographique et audiovisuel de leur époque. En s'appuyant sur une approche systématique, elle est applicable au corpus des films utilitaires médico-sanitaires français que nous avons réunis. D'autre part, elle inspire une trame d'analyse structurée par deux types de comparaisons : synchrones / diachrones et nationales / internationales. La deuxième démarche, qui figure au centre des travaux internationaux récents s'adosse aux travaux concernant « le tournant iconographique » des sciences du début du $20^{\mathrm{e}}$ siècle, c'est-à-dire la promotion des différentes formes de représentation et d'image ${ }^{60}$ dans l'établissement d'une preuve en sciences. ${ }^{61}$ Ces travaux soulignent le développement des méthodes graphiques comme dispositifs pour enregistrer, inscrire et mettre en scène des phénomènes corporels et naturels, et de ce fait produire ce qui est alors considéré comme une nouvelle « réalité » scientifique : visuelle, indépendante et « objective ». Bien que l'interrogation sur le statut des images dans la production du savoir scientifique soit devenue une préoccupation de la recherche, peu d'études étendent ces analyses à la circulation des images animés depuis les sphères d'expertise scientifique vers la société. ${ }^{62}$ Et encore moins de travaux s'intéressent au rôle joué dans

\footnotetext{
${ }^{59}$ Matthes, 1992, pp. 75-99.

60 Pour le « iconic turn » en histoire des sciences, voir : Cartwright, 1995. Fox, Lawrence, 1988. Gilman, 1995. Jordanova, 1990, pp. 89-99. Maehle, 1993, pp. 563-586. Sappol, 2004, pp. 1-6, 37-41.

61 Daston, Galison, 2007. Martinet, 1994. Thévenard, Tassel, 1948.

62 Daston, Galison, 2007. Verdicchio, 2010.
} 


\section{SHS Web of Conferences}

cette circulation par le média du film utilitaire. ${ }^{63}$ Cette démarche s'inscrit dans le courant plus large des « visual studies » et des « digital humanities ». 64

Concrètement, le projet de recherche MedFilm ${ }^{65}$ a d'abord exploré comment un certain nombre d'institutions et d'organisations médicales et sanitaires à partir de l'entre-deux guerres et à échelle internationale se sont saisis du film pour promouvoir leurs programmes d'éducation à la santé. Une telle approche implique de s'intéresser à l'intégralité de la chaine, depuis la production et diffusion de ces films jusqu'à leur réception par les publics visés. ${ }^{66}$ Puisque la période de l'entre-deux-guerres était mieux connue que celle de 1945 à 1960, nous avons investi celle-ci à partir de fonds de films destinés à l'éducation sanitaire à l'école ${ }^{67}$, puis un corpus de films issus d'une société de production de films médicaux particulièrement importante, attachée à la figure de son principal réalisateur, Éric Duvivier. ${ }^{68}$

63 Reichert, 2007.
64 Voir à titre d'exemple la plate-forme expérimentale «culture visuelle », média social d'enseignement et de recherche, qui
se présente à la fois comme un organe de publication, une communauté académique et un projet de recherche. Cette plate-
forme expérimentale est réalisée par le Laboratoire d'histoire visuelle contemporaine (Lhivic.EHESS) sous la direction d'André
Gunthert. Ainsi que Digital Humanities Laboratory (DHLAB) à l'Université de Lausanne créé en 2012 sous la direction de
Frédéric Kaplan.

65 Pluridisciplinaire, situé aux frontières entre histoire, histoire des sciences et des techniques et sciences de l'information et de la communication le programme de recherche est porté depuis trois ans par deux équipes de recherches, l'une en histoire des sciences et techniques, le Département d'Histoire des Sciences de la Vie et de la Santé (DHVS) au sein de l'UMR Sociétés, Acteurs, Gouvernements en Europe (SAGE, UMR 7363), l'autre en sciences de l'information et de la communication, le Centre de Recherche sur les Médiations (CREM, EA 3476). Au sein du laboratoire SAGE, les enseignants-chercheurs en histoire des sciences et des techniques impliqués sont Christian Bonah, Anne Rasmussen, Marion Thomas, Alexis Zimmer, Valentine Hoffbeck, Nils Kessel et Joël Danet, ce dernier recruté sur le projet en tant qu'ingénieur de recherche. Pour l'équipe du CREM, les enseignants chercheurs spécialistes en sciences de l'information et de la communication sont Vincent Lowy, Anne Masseran et Emmanuelle Simon ainsi que France Garat recruté sur le projet en tant qu'ingénieure de recherche. Les recherches s'effectuent en association avec des chercheurs en histoire contemporaine de l'équipe Arts, Civilisation et Histoire en Europe (ARCHE, EA 3400) de l'Université de Strasbourg, notamment Alexandre Sumpf qui est maître de conférences en histoire contemporaine de l'Europe centrale et orientale à l'UFR des Sciences historiques de l'Université de Strasbourg, spécialiste de l'histoire du film documentaire et membre junior de l'IUF. Au Laboratoire de recherche historique Rhône-Alpes (LARHRA, UMR 5190) à l'université Lumière Lyon 2 avec Isabelle von Bueltzingsloewen qui est professeur en histoire contemporaine spécialiste de l'histoire de l'Allemagne et depuis quelques années s'intéresse plus particulièrement à l'histoire de la psychiatrie, de l'assistance aux malades mentaux en France au $20^{\mathrm{e}}$ siècle et à l'histoire de l'éducation pour la santé. Enfin avec Thierry Lefebvre (voir note 23) et Richard Millet au Centre d'Etude et de Recherche interdisciplinaire Lettres, Arts et Cinéma (CERILAC) à l'UFR Sciences du Vivant (secteur «Sciences Médias Sociétés ») de l'Université Paris Diderot.

66 Colloque d'ouverture les 26 et 27 mai 2011 à la Fondation Brocher à Genève « Communicating good health : movies, medecine, and the cultures of risk in the 20th Century ». Voir pour une présentation, les papiers précirculés, un compte rendu et des extraits de films (avec mot de passe) : http://medfilm. misha. fr consulté en date du 06/05/2014. Les actes du colloque sont actuellement en voie de publication sous la forme d'une monographie à paraître à Rochester University Press.

${ }^{67}$ Une première journée d'études a été organisée le vendredi 20 janvier 2012 sur le thème « À la croisée de l'orientation professionnelle et de l'éducation sanitaire à l'école. Les films du Scérén-CNDP » aux Grands Moulins de l'Université Paris-Diderot organisée par Christian Bonah et Thierry Lefebvre. La thématique de cette journée a été adaptée en fonction des résultats de notre travail de recherches en archives et notamment sur le fond du CNDP. Pour une présentation et le programme voir la rubrique « recherche » du site MedFilm : MedFilm - Journée d'étude n¹, « À la croisée de l'orientation professionnelle et de l'éducation sanitaire à l'école. Les films du SCÉRÉN-CNDP ». http://medfilm. unistra.fr/s/recherche consulté en date du 06/05/2014. Nous remercions Laurent Garreau du SCERENCNDP pour la collaboration établie. Les résultats ont été présentés par une publication dans Newsletter (électronique) du CNDP en Février 2012. http: //www. cndp.fr/media-sceren/lettreinfo/index04.php consulté en date du 06/05/2014.

68 Cette deuxième journée d'études « À la croisée de la promotion, de la formation et du film expérimental médical : Éric Duvivier et la compagnie de production ScienceFilm (1950-2000) » a été consacrée le 21 septembre 2012 à la thématique des techniques audiovisuelles de persuasion et transmission d'un message médico-sanitaire par des images. Elle a été centrée sur la société de production de films «ScienceFilm » et son réalisateur Éric Duvivier. Pour la présentation et le programme voir : http://medfilm. unistra.fr/s/recherche consulté en date du 06/05/2014. A partir de cette manifestation, et compte tenu du peu d'informations disponibles sur les films présentés, nous avons engagé le parti pris d'enregistrer visuellement nos journées d'études et de les rendre accessibles sous forme de vidéos sur le site de MedFilm. Cette captation est le fruit d'une collaboration avec le CERIMES dont les responsables et tout particulièrement Peggy Domeyne soient remerciés. Les vidéos des interventions sont accessibles sous http://medfilm.unistra.fr/s/recherche consulté en date du 06/05/2014 et directement au CERIMES : http://www.canal-u.tv/producteurs/cerimes/les_films_realises_par_eric_ duvivier/journee_medfilm_eric_duvivier consulté en date du 06/05/2014. 
Ce dernier type de films, par leur caractère hybride, entre art visuel pur et information scientifique, nous a ensuite amené à nous interroger sur les liens que les films médico-sanitaires utilitaires pouvaient tisser avec le milieu de la création cinématographique. ${ }^{69}$ Le souci de nous donner une perspective historique plus profonde nous a conduit à revenir vers la figure de Jean Benoit-Lévy, principal cinéaste hygiéniste de l'entre-deux-guerres, période par ailleurs propice à une comparaison franco-allemande. ${ }^{70}$ Enfin, la conférence finale du projet de recherche a tenté de produire une première synthèse partielle de l'histoire des films utilitaires médicaux et sanitaires en France entre 1895 et $1960 .^{71}$

Sur la base de ces premiers travaux, le projet MedFilm s'est orienté vers une recherche plus systématique. Nous avons d'abord constitué une filmographie à partir d'une revue systématique des périodiques médicaux ${ }^{72}$ et de cinéma ainsi qu'à partir d'anciens catalogues de films. Ce chantier s'est avéré plus long que prévue et sa réalisation a permis de mettre à jour une quantité de films bien supérieure à ce que nous avions initialement envisagé (250-500 films). ${ }^{73}$ Devant ce résultat positif mais non sans problèmes, une nouvelle difficulté consistait à envisager des stratégies de recherche pour traiter ce nombre de films supérieur à nos attentes et pour établir des priorités d'exploitation. Nous avons opté pour une approche par institutions détentrices, ce qui possède l'avantage de faciliter le traitement des questions de localisation et de l'obtention de droits de diffusion des films identifiés. ${ }^{74}$ Actuellement, nous disposons de plus de 250 films numérisés avec accord de leur ayants-droits pour leur mise à disposition intégrale sur la plate-forme MedFilm. Leur description et analyse est progressivement intégrée à la plate-forme. A l'heure actuelle, elle comporte une centaine de fiches analytiques associées aux films correspondants. ${ }^{75} \mathrm{~A}$ partir de films individuels particulièrement marquants et d'ensembles

${ }^{69}$ Cette troisième journée d'étude «Style, motifs et expérimentations : Eric Duvivier cinéaste » a eu lieu à Nancy le 3 juin 2013, à l'Institut Européen de Cinéma et d'Audiovisuel (Université de Lorraine). Organisée par Vincent Lowy, France Garat et Emmanuelle Simon, en collaboration avec le Conservatoire Régional de l'Image Nancy-Lorraine qui détient une grande partie du fonds Éric Duvivier, la journée s'est intéressé au contexte cinématographique des films médicaux et sanitaires utilitaires. Pour la présentation et le programme voir : http://medfilm. unistra.fr/s/recherche consulté en date du 06/05/2014. La publication des interventions de ces deux journées est programmée sous la direction de Thierry Lefebvre et Christian Bonah aux éditions Le Bord de l'Eau dans la collection Ciné-politique.

${ }^{70}$ Le workshop international franco-allemand « Missionary \& commissioned. Medical film directors and their oeuvre in francogerman comparison. Jean-Benoit Lévy - Nicholas Kaufmann - Kurt Thomalla » consacré à l'étude comparée des productions de trois réalisateurs importants de films de prévention s'est tenu à Strasbourg les 13 et 14 mai 2013 en partenariat avec le Max Planck Institut für Bildungsforschung (Berlin) et l'INA-France, complète le tableau des réalisations scientifiques du projet. Pour la présentation et le programme voir : http://medfilm. unistra.fr/s/recherche consulté en date du 06/05/2014. Les vidéos des interventions sont accessibles sous http://medfilm. unistra.fr/s/mprogramme consulté en date du 06/05/2014.

${ }^{71}$ La conférence internationale « Information, persuasion, propagande. Le film médico-sanitaire comme mode de communication entre science, médecine, sphère publique et société en France, 1895-1960 » a eu lieu les 20 et 21 juin 2014 à Strasbourg. Voir pour une présentation, l'appel à communication, les résumés, un compte rendu et une captation du colloque http://medfilm. unistra.fr/s/recherche consulté en date du15/07/2014.

72 Les articles analysés de manière systématique entre 1895 et 1960 dans les périodiques médicaux et scientifiques français concernent la Presse Médicale, Concours médical, Semaine médicale, Bulletin de l'Académie de médecine, Gazette médicale de Strasbourg, C.R. Société de Biologie, C.R. Académie des Sciences, Revue neurologique, Bulletin de la Société d'Obstétrique et de Gynécologie, Strasbourg Médical, la presse sanitaire La Vie saine, Revue internationale du cinéma éducateur, la presse cinématographique Cinémagazine, Phono-Ciné-Gazette, Revue du cinéma des thèses de médecine ainsi que catalogues de films (Catalogue des films d'enseignement scientifiques et médicaux ininflammables, Paris, Pathé-Enseignement, s.d. [1924], Catalogue Pathé-Baby, Paris, Pathé-Cinéma, 1930, etc.

${ }^{73}$ La filmographie sera accessible à la fin du programme de recherche sur le site MedFilm.

${ }^{74}$ A partir de ce point nous avons mené la négociation avec deux institutions significatives détentrices de films pour aboutir à la mise à disposition et de numérisation. Une convention de collaboration a été signé avec le CNDP d'une part, avec le CERIMES d'autre part. Des discussions pour une convention avec d'autres institutions est engagée notamment pour les fonds importants de l'INA-Ministère Agriculture, de l'Institut Pasteur et de l'ECPAD. Par ailleurs nous avons préparé et signé une convention avec les ayants-droits de deux réalisateurs majeurs pour les films utilitaires médico-sanitaires français concernant l'œuvre de Jean BenoitLévy et d'Éric Duvivier. Voir : http://medfilm.unistra.fr/wiki/Cat\%C3\%A9gorie:Fiches_valid\%C3\%A9es consulté en date du 06/05/2014.

75 L'ouverture officielle de la plate-forme a été annoncée lors du Colloque national du collège des enseignants de SHS en médecine les 21-23 juin 2013 à Amiens, une réunion biannuelle à mi-chemin entre recherche, enseignement et recherche sur des dispositifs d'enseignement innovants pour les SHS en médecine. Pour le collège voir : http: //collegeshsenmedecine. 
cohérents identifiés au sein de la plate-forme nous avons engagé des communications ${ }^{76}$ et des publications ${ }^{77}$, souvent collaboratives, en prolongement des manifestations scientifiques présentées au préalable. Leurs thématiques et leurs questions de recherche sont souvent directement stimulées par les préparations et les tenues des enseignements par lesquels le projet de recherche est né et pour lesquels il continue à servir.

\section{Des contenus de recherche à l'outil d'enseignement : la plate-forme mediawiki MedFilm}

Depuis plus de dix ans, pour préparer nos enseignements, nous cherchons des films utilitaires concernant la médecine et la santé dans une définition large. Pourquoi ne pas les rassembler et les mettre en commun ? Cette interrogation a initié la conception d'un outil intégré enseignement-recherche sur les films utilitaires médicaux et sanitaires. Sans données contextualisantes, de tels films restent inintelligibles, par conséquent impossible à manier. Puisque nous menons depuis des années des recherches dans ce domaine, pourquoi ne pas les formaliser ? Pour répondre à ces deux observations nous avons élaboré dans le cadre du programme de recherche MedFilm une démarche et un outil.

Aux différents films identifiés par l'inventaire sont associées des fiches individuelles d'identité et d'analyse. Construites selon une trame analytique formalisée,elles donnent accès aux caractéristiques principales des films. ${ }^{78}$ Outre leurs caractéristiques techniques, les fiches indiquent leurs synopsis et les situent en fonction de leur thématique, de leur aspect technique, de leur chronologie ou encore de leur intérêt d'un point de vue pédagogique. Les analyses sont produites par les chercheurs du projet MedFilm, et de plus en plus par les étudiants des enseignements en rapport. Leur publication sur la plate-forme est soumise à une validation par les membres de l'équipe selon une grille d'analyse validée lors du séminaire bimestriel du projet. Par ailleurs, des documents annexes aux différentes fiches incluent des sources originales et des publications secondaires concernant les films. ${ }^{79}$

L'objectif principal consistant à fournir un accès libre et pratique à ces documents pour une large population de chercheurs, enseignants et étudiants, les choix technologiques de la plate-forme se composent de deux ensembles : un site web classique d'une part, qui accueille les informations générales sur le projet et les annonces et résultats de notre recherche, la base de données d'autre part, qui prend la forme d'un MediaWiki $?^{80}$ MediaWiki est un ensemble wiki à base de logiciels libres

etud.univ-montp1.fr consulté en date du 06/05/2014. Pour le colloque du collège à Amiens en 2013 : http://collegeshsenmedecine.etud.univ-montp1.fr/congres-et-colloques/congres-2013 consulté en date du 06/05/2014.

76 A titre d'exemple : La représentation du handicap mental dans les films de propagande hygiéniste nazis, conférence donnée par Christian Bonah et Vincent Lowy dans le cadre du séminaire du Comité scientifique de l'éthique, des pratiques professionnelles et de l'évaluation du Groupement national des établissements sociaux publics (GEPSo), Annecy, 23 octobre 2010. Anne Masseran (CREM, université de Strasbourg), Emmanuelle Simon (CREM, université de Lorraine), Les films sanitaires et leurs publics, Communication présentée au séminaire de recherche de l'équipe Praxis du CREM, 22 ai 2012. Emmanuelle Simon (CREM, université de Lorraine), Figures du soin dans le cinéma médical, Communication présentée au séminaire de recherche de l'équipe GreCSS, 31 mai 2012.

77 Bonah, Lowy, 2013, pp. 46-56. Bonah, Danet, 2012, pp. 274-282. Bonah, 2012, p. 151-172. Bonah, 2011, pp. 152-175. Bonah, Lowy, 2010, pp. 35-49. Bonah, Zimmer, 2009, pp. 87-106. Bonah, Laukötter, 2009, 121-145.

78 Voir pour les détails les fiches individuelles : http://medfilm.unistra.fr/wiki/Cat\%C3\%A9gorie:Fiches_valid\% C3\%A9es consulté en date du 06/05/2014.

79 La plate-forme, MedFilm héberge également des reproductions d'archives écrites, relatives aux films - dossiers de productions, articles de presse, communications de journées d'études - permettant de contextualiser les films. Les documents sont associés par un lien Documents attachés à la fiche du film concerné.

${ }^{80}$ La plate-forme pédagogique MedFilm à été élaborée en partenariat avec la Direction des Usages numériques de l'Université de Strasbourg. L'équipe MedFilm tient ici à remercier en particulier le responsable de la DUN, Philippe Portelli, ainsi que ses collaborateurs David, Gauckler, Ludovic Strappazon et Vincent Couturier pour leur aide précieuse. La mise à disposition des films sur la plate-forme MedFilm est rendu possible par une série de conventions de partenariats avec le CNDP, le CERIMES, etc. Ainsi que des conventions avec des ayants-droits des réalisateurs de films : Madame et Monsieur Éric Duvicier, Madame Liliane Jolivet. 
open source, développé à l'origine pour wikipédia et aujourd'hui utilisé par plusieurs autres projets. Les membres de la Direction des Usages Numériques de l'Université de Strasbourg l'ont adapté aux besoins spécifiques de MedFilm. ${ }^{81}$ Le MediaWiki MedFilm remplit deux fonctions : le stockage, qui implique la collecte, la numérisation et la sélection raisonnée films médico-sanitaires devenus disponible via le réseau internet. La seconde fonction est didactique. Elle vise principalement à faciliter l'usage de ces films, par la classification catégorielle de ces derniers, par la construction d'une architecture favorisant leur emploi et par l'élaboration de propositions thématiques d'enseignement (kits pédagogiques) qui sont issues de préparations de cours et s'apparentent à des collections de «syllabi » connus outre Atlantique. ${ }^{82}$ Les groupements thématiques proposés sont évolutifs, enrichis progressivement et ouverts à proposition au-delà de notre équipe de recherche. Les premiers sont : «l'hôpital », « les professions médicales », «portraits de patients », « la fin de vie », ou encore « le médicament ». Les kits sont essentiellement constitués de documents informatifs, accompagnés de références et de trames pédagogiques. Ils comportent en principe un conducteur de cours, des liens vers les fiches descriptives et analytiques des films employés et des documents écrits (articles de journaux, extraits d'ouvrages, textes d'interventions universitaires, etc.). A noter que dans le cadre des kits, le projet souhaite, à l'instar de la New York «Literature, Arts \& Medicine Database », élargir le champ cinématographique à des disciplines culturelles et artistiques. A titre d'exemple, le cours "Cinéma, littérature et médecine » dispensé à la Faculté de médecine de Strasbourg, résulte d'une démarche liée à la construction du kit, qui confronte des documents littéraires, médiatiques, voire musicaux, autour du même sujet sanitaire en faisant percevoir leur complémentarité d'approches.

La ressemblance de la plate-forme avec l'encyclopédie wikipedia, dont elle partage la structure, est volontaire pour faciliter son utilisation et pour suggérer l'aspect collaboratif de notre projet. La différence essentielle consiste dans le fait que les informations contenues sur la plate-forme sont validées de manière académique avant leur publication. De même, tout changement doit passer obligatoirement par une validation auprès des responsables éditoriaux de MedFilm. Comme pour l'encyclopédie wikipedia, la plate-forme disposera à terme d'une structure de renvois internes plutôt que d'une structure hiérarchisée de l'information permettant la circulation entre différentes informations via des catégories et des mots clés. La plate-forme est associée à un système de gestion créateur de livres et d'exportation des fiches et des documents sous forme PDF/ODF.

Une dernière difficulté à résoudre pour la constitution de la plate-forme concerne les conditions de la mise à disposition des films par les archives et cinémathèques détenteurs. Seul un accès gratuit et restreint au monde de l'enseignement et de la recherche a permis de parvenir à la signature des conventions conditionnant leur mise à disposition. A cet effet, la plate-forme est couplée, pour le visionnage intégral des films, au système d'identification «shibbolette ». Il s'agit d' un mécanisme de propagation d'identités, développé par le consortium Internet 2 qui regroupe 207 universités et centres de recherches. ${ }^{83}$ La connexion nécessite une adresse e-mail universitaire avec identifiant et mot de passe.

${ }^{81}$ MediaWiki est un ensemble wiki à base de logiciels libres Open source, développé à l'origine pour Wikipédia et aujourd'hui utilisé par plusieurs autres projets de l'association à but non lucratif Wikimedia Foundation ainsi que par beaucoup d'autres wikis. L'utilisation et l'adaptation de cette ressource pour les besoins du projet MedFilm à été suggéré et réalisé par Ludovic Strappazon à la Direction des Usages Numériques de l'Université de Strasbourg. Nous le remercions pour les innombrables moments d'échange cordiaux et constructifs. Pour mediawiki voir : http://www. mediawiki .org consulté en date du 06/05/2014.

82 Pour une initiative comparable concernant l'enseignement en l'histoire de la médecine voir la base de données de la National Library of Medecine, History of Medicine Division qui rassemble des programmes de cours (syllabi) : http://www.nlm.nih.gov/hmd/collections/digital/syllabi consulté en date du 06/05/2014.

83 La délégation de l'authentification réutilise les techniques de Single Sign-On web (redirection, cookies, ....). Lors de l'accès initial à une ressource numérique, l'utilisateur est redirigé vers le service de découverte de la fédération, d'où il sélectionne son établissement d'origine ; il est ensuite renvoyé vers son fournisseur d'identités. Le pré-requis pour le fournisseur d'identités est de disposer d'un service d'authentification global tel que Central Authentication Service. En France Shibboleth est porté par la fédération Éducation-Recherche RENATER. 


\section{Retours pédagogiques : le projet formation MedFilm}

L'avancée de nos recherches et la réalisation concrète de la plate-forme nous ont incité enfin à revenir vers les débuts de notre cheminement, à savoir les préoccupations pédagogiques pour l'enseignement des SHS en médecine et pour les études sociales des sciences. A l'image d'une spirale, ce trajet implique qu'un retour à l'identique s'accompagne de déplacements. Basé sur le projet de recherche nous avons rédigé et soumis un projet d'enseignement innovant à un appel d'offre Idex-formation de l'Université de Strasbourg. ${ }^{84}$ Les enseignements proposés dans ce cadre entendent intégrer la dimension de recherche et favoriser l'utilisation de la plate-forme à des niveaux et sous formes multiples.

Dans un premier volet, le projet formation MedFilm permet depuis la rentrée 2013-2014 la tenue d'une classe renversée pour la filière complète des étudiants en 1ere année d'études de santé (PACES). Dans le cadre de l'unité d'enseignement «Santé, société, humanités » (UE 7) le projet propose en extension des TD classiques actuels la mise à disposition des supports pédagogiques audio-visuels via la plate-forme associant trois éléments : (1) la mise à disposition de documents audio-visuels (elearning/devoir maison), (2) l'application et explicitation des analyses SHS en TD (présentiel), (3) l'espace de discussion sous la forme d'un blog ouvert pour un temps limité pendant la mise en place des $\mathrm{TD}$, favorisant une discussion entre étudiants au-delà du présentiel $\mathrm{TD}$, et des échanges avec l'enseignant entre les séances de TD.

Ceci a donné lieu au recrutement d'un post-doctorant ${ }^{85}$ qui, en collaboration avec l'ATER chargé des enseignements, et le responsable des enseignements magistraux, a conçu un environnement pédagogique $^{86}$ lié à la plate-forme pour enrichir les TD de SHS en PACES et accompagner les étudiants. ${ }^{87}$ Les travaux dirigés portent en 2013-2014 sur l'analyse critique de la contraception, analyse qui s'ancre dans la lecture d'un film-documentaire de 1970 La contraception moderne que les étudiants sont invités, via la plate-forme, à visionner avant le début des séquences de TD. Le film invite à envisager la contraception comme une pratique indissociablement sociale et médicale, dont il s'agit d'étudier chaque aspects. Son exemple sert à mieux comprendre les relations complexes entre ce que l'on appelle souvent, en les dissociant trop strictement, «science» et « société ». Il s'agit d'interroger et de problématiser des visions simples voire simplistes, mises en scène par le film, aussi considérées comme des « lieux communs », afin de permettre à l'étudiant de mieux comprendre les enjeux multiples et souvent complexes des professions de santé. Plus précisément, le TD les invite à considérer de manière plus large : d'une part les stratégies à l'œuvre pour gouverner les populations en encadrant les modes contraceptifs, d'autre part les problèmes liés aux effets dits secondaires des technologies pharmaceutiques. Et ceci autrement que par les catégories de : «progrès », « soins désintéressés », « tragédies », « tributs au progrès » ou encore de « risque ». Notre objectif consiste donc à complexifier la contraception par l'histoire. Plutôt que de privilégier le jugement moral ou la vision normative, elle est tantôt considérée, selon le point de vue politique des uns et des autres, comme une pratique bénéfique, ou au contraire "démoniaque », nous en proposons une compréhension critique. En tant que pratique sociale historiquement déterminée, nous supposons qu'elle est susceptible de nous renseigner sur les manières dont on produit du pouvoir en société, sur les manières dont la vie est

\footnotetext{
${ }^{84}$ Le projet de formation MedFilm est soutenu pendant la période 2013 à 2015 dans le cadre des appels à projets « Idex Enseignement » de l'Université de Strasbourg.

85 Alexis Zimmer a été recruté à cet effet. La conception et la préparation de ce contenu de cours est essentiellement rédigée par lui et par Nils Kessel, ATER, dans le cadre de leur mission d'enseignement et de recherche.

86 Il se trouve sur la plate-forme Moodle : https : / /ent. unistra.fr consulté en date du 06/05/2014 sous la rubrique « Travaux dirigés - Sciences-humaines en médecine - PACES ». Cet environnement d'apprentissage constitue également un lieu d'échange avec le corps enseignant, puisqu'un forum permet à l'étudiant d'adresser des questions, des interrogations ou des critiques, liées aux difficultés et incompréhensions que suscite cet enseignement.

${ }^{87}$ La page des documents supplémentaires inclut des émissions radiophoniques récentes, des liens vers des vidéos d'archives de l'Institut National de l'Audiovisuel (INA) et des sites web pour aider les étudiants à mettre en œuvre la posture critique déployée lors de l'enseignement.
} 
gouvernée et sur l'intérêt de réguler le danger par le « risque ». Afin de permettre aux étudiants de mettre en œuvre ce questionnement, nous proposons une structure argumentative scandée par quatre grandes interrogations : (1) Qu'est-ce que la contraception ? (2) En quoi la contraception est une activité «scientifique»? (3) Pourquoi y a-t-il des controverses sur la contraception et ses moyens ? (4) Qu'est-ce que ceci nous apprend sur les études de santé ? Ces quatre questions correspondent aux quatre aspects qui seront successivement analysés en TD : (1) La contraception, une pratique déterminée historiquement ; (2) La contraception comme fenêtre pour aborder la compréhension et la production scientifique des corps humains ; (3) La controverse sociotechnique comme le produit d'une confrontation entre diverses formes de gouvernement (des sexualités, des naissances, des corps) et divers type d'acteurs, de sujets et d'intérêts ; et enfin (4) L'importance d'une réflexion par les - futurs - professionnels de la santé sur l'action normative et normalisante de leur travail et la nécessaire distance critique à entretenir face aux récits glorifiants d'une science « neutre ».

Le projet formation MedFilm s'est appuyé sur la plate-forme dans un autre contexte : son deuxième volet interdisciplinaire concerne les étudiants en DCEM 1-3 en médecine ainsi qu'en troisième cycle en médecine générale et dans le cadre d'enseignements d'ouverture mutualisés avec les formations en Master. ${ }^{88}$ L'ensemble des formations de ce deuxième volet se caractérise par une composante « enseignement par et pour la recherche » significative, notamment par le mode d'évaluation de ces unités d'enseignement qui se fait sous forme d'exposés et de «mini mémoires de recherche ». Ces travaux, en fonction de leur pertinence, sont en retour intégrés dans la plate-forme sous la forme de fiches analytiques du MediaWiki MedFilm après modération et validation universitaire. L'interdisciplinarité des projets et propositions de formation relève autant du contenu des enseignements proposés (contenu et/ou collaboration entre enseignants de disciplines différentes) que de la diversité des origines des étudiants concernés (médecine, pharmacie, sciences de la vie, histoire, histoire des sciences, sociologie, sciences de l'information et de la communication). Cette hétérogénéité des publics enseignés secrète une dynamique propre, palpable par les échanges pendant les cours. Elle favorise une co-construction et un co-apprentissage entre étudiants dont la conception pédagogique du projet souhaite tirer tout son avantage. A titre d'exemple, prenons les modalités de l'unité d'enseignement "Propagande, information, communication au XXe siècle ». Elle mutualise le Master 1 et Master 2 de la Faculté des Sciences historiques, et le Master 2 Sciences et Société - Histoire, philosophie et médiation des sciences de la Faculté des sciences économiques et gestion. Assurée conjointement par un enseignant de l'UFR Histoire (Alexandre Sumpf) et de l'UFR Médecine (Christian Bonah), adossée aux projets de recherche ANR MedFilm et au projet Interreg RhinFilm ${ }^{89}$ l'unité d'enseignement comporte une forte composante recherche sur les fonds d'archives et l'histoire du film « utilitaire ». Sa validation se fait sous forme de dossiers de recherche qui peuvent concerner ou bien l'analyse d'une série de films, avec la rédaction de leurs fiches correspondantes pour la plate-forme MedFilm, ou bien une démarche de

\footnotetext{
88 A titre d'exemple : EC 03 Cinéma, littérature et médecine I : Portraits de patients (PCEM2 et DCEM1), mutualisé avec le Master de Communication scientifique et le Master Sciences et Société - Histoire, philosophie et médiation des sciences: effectif 40 étudiants M1 / M2. MO 04 Cinéma, Littérature et médecine II : L'hôpital et ses représentations : soins, vie, mutualisé avec le Master Sciences et Société - Histoire, philosophie et médiation des sciences et en lien avec l'UE Sociologie de l'hôpital (Master Sciences Sociales, spécialité Sociologie) : effectif 40 étudiants M1. UE ouverture Master Neurosciences : Histoire et épistémologie des neurosciences, mutualisée avec le Master Sciences et Société - Histoire, philosophie et médiation des sciences : effectif 35 étudiants en M2. UE d'ouverture professionnelle Master Sciences du médicament, Spécialité Pharmacologie, «L'innovation thérapeutique en perspective historique : sciences, technologies et société », mutualisée avec le Master Sciences et Société - Histoire, philosophie et médiation des sciences : effectif 30 étudiants en M1/M2. UE « Propagande, information, communication au XXe siècle » mutualisée entre le M1 et M2 de la Faculté des Sciences historiques et le M2 du Master Sciences et Société - Histoire, philosophie et médiation des sciences de la Faculté des sciences économiques et gestion : création d'UE interdisciplinaire autour du projet ANR MedFilm et du projet Offensive Sciences RhinFilm dans le cadre du quadriennal 20132017. Première ouverture en septembre 2013. Cette UE comporte une forte composante recherche sur les fonds d'archives et l'histoire du film « utilitaire».

${ }^{89}$ Voir pour une présentation : http://rhinfilm. unistra.fr consulté en date du 06/05/2014.
} 
recherche pour identifier, décrire et localiser un fond de films utilitaires original. Ces dossiers remis sont relus par l'équipe de recherche MedFilm avant leur intégration dans la plate-forme.

Dans le principe d'associer étroitement les activités de recherche et d'enseignement, les formations évoquées peuvent donner lieu à des travaux de recherche au niveau Master 2, et préparer à des travaux de thèse en histoire ou en histoire des sciences sur des corpus de films intégrés ou susceptibles d'intégrer la plate-forme MedFilm. Celle-ci tire ainsi bénéfice des apports des nouvelles technologies décrits plus haut, avec la possibilité d'intégrer les travaux des étudiants dans le contenu même de la plate-forme collaborative. Les sujets sont identifiés et choisis à partir de la disponibilité des supports de films dans la plate-forme MedFilm et les travaux des étudiants seront immédiatement valorisés par leur réintégration dans la plate-forme sous forme de fiches et de documents intégraux qui portent leur signature.

Les mois à venir seront consacrés à la préparation d'un cours en ligne ouvert et massif (Massive open online course MOOC) en lien avec la plate-forme pédagogique et de recherche MedFilm. Son sujet et son périmètre sont à l'étude actuellement. En collaboration avec la Direction des usages numériques (DUN) nous souhaitons pousser l'expérimentation d'une interaction plus aboutie avec le public. Pour cela, nous envisageons d'ouvrir trois axes d'échanges sur la plate-forme : (1) l'ouverture des pages de discussion associées aux films ; (2) la mise à disposition de fiches vierges et d'ébauches soumises à la rédaction d'autres contributeurs que l'équipe d'enseignants-chercheurs MedFilm ; ainsi que (3) la sollicitation du public pour la recherche d'éléments rares dans une perspective de sauvegarde et de valorisation patrimoniale des films utilitaires médicaux et sanitaires.

\section{Conclusion}

Conçue dans le cadre du projet de recherche ANR «Instruire, informer, communiquer, éduquer : Le film médico-sanitaire en France, 1895-1960 » la plate-forme numérique interactive MedFilm vise à articuler la mise en ligne d'archives de films médicaux et sanitaires du $20^{\mathrm{e}}$ siècle avec leurs analyses écrites. Issue d'une préoccupation pédagogique, devenue projet de recherche, la plate-forme trouve aujourd'hui son application dans l'enseignement, qu'il s'agisse de grands effectifs (PACES) sous forme de « classes renversées » associant les humanités digitales à l'enseignement classiques sous forme de travaux dirigés présentiels et de cours magistraux, ou d'enseignements plus spécifiques pour le niveau Master. La présentation a cherché à revenir sur le cheminement de ce projet collaboratif à orientation interdisciplinaire, proposant une approche intégrée enseignement-recherche ouvrant à des formes multiples d'une formation par et pour la recherche.

Les conférences et workshops internationaux ont participé à la constitution d'un réseau international de recherche sur notre thématique dont nous espérons tirer profit pour la poursuite de nos travaux. La fin du financement du projet en septembre 2014 nécessite actuellement la préparation de nouveaux projets de recherches à soumettre sur le plan national et international afin de pérenniser et de poursuivre le travail engagé.

\section{Références}

Belhoste, Bruno, Gispert, Hélène, Hulin, Nicole (éds.), Les sciences au Lycée. Un siècle de réformes des mathématiques et de la physique en France et à l'étranger. Paris, Vuibert \& INRP, 1996.

Belting, Hans Pour une anthropologie des images, traduit de l'allemand par J. Torrent, Paris, Gallimard, 2004.

Billroth, Theodor, Über das Lehren und Lernen der medicinischen Wissenschaften an den Universitäten der deutschen Nation, Wien, 1876.

Blasco, P.,"Literature and Movies for Medical Students", Family Medicine, 33, 2001, pp. 426-428.

Boisaubin, E.V. et Winkler, M.G., "Seing Patients and Life Contexts: The Visual Arts in Med Education”, The American Journal of Medical Science, 319, 2000, pp. 292-296. 
Bonah, Christian ; Danet, Joel, «Transmettre à et pour des enfants en détresse. Observations à partir de films institutionnels autour de la 2ème guerre mondiale », Neuropsychiatrie de l'enfance et de l'adolescence 60, 2012, pp. 274-282.

Bonah, Christian ; Lowy, Vincent, «Lorsque le regard l'emporte sur le message. Les courts-métrages de lutte contre la tuberculose d’Edgar G. Ulmer », Special Issue « Cinéma et science », Alliage, N71, hiver 2012-2013, pp. 46-56.

Bonah, Christian ; Rasmussen, Anne ; (éds.), Actes du colloque : 10 ans d'enseignement de sciences humaines et sociales en médecine, Faculté de Médecine de Strasbourg, Strasbourg, 2005.

Bonah, Christian, «Une introduction à l'étude de la médecine expérimentale à l'ère de la reproduction mécanique : un legs de Claude Bernard et / ou d'Etienne Jules Marey ? » in Barbara, Jean-Gaël ; Corvol, Pierre, Les élèves de Claude Bernard. Les nouvelles disciplines physiologiques en France au tournant du XXe siècle, Paris, Hermann, 2012, p. 151-172.

Bonah, Christian, " "Health crusades" : Environmental approaches as public health strategies against infections in sanitary propaganda films, 1930 - 1960", in Berridge, Virginia; Gorsky, Martin, Environment, Health and History, London, Palgrave Macmillan, 2011, pp. 152-175.

Bonah, Christian, "L'enseignement des sciences humaines et sociales en médecine : éléments pour un panorama de la situation en France », in Académie Suisse des sciences humaines et sociales, Médecine et sciences humaines. Sciences humaines en médecine : formation et collaboration, Berne, Schweizerische Akademie der Geistes und Sozialwissenschaften, 2006, pp. 9-18.

Bonah, Christian, «Propositions pour une utilisation du septième art pour enseigner les relations entre science, médecine et société », in Bonah, Christian ; Rasmussen, Anne ; (éds.), Actes du colloque : 10 ans d'enseignement de sciences humaines et sociales en médecine, Faculté de Médecine de Strasbourg, Strasbourg, 2005, pp. 81-84.

Bonah, Christian, Haxaire, Claudie, Mouillie, Jean-Marc, Penchaud, Anne-Laurence, Visier, Laurent (éds.), Médecine, santé et sciences humaines. Manuel du Collège des enseignants de SHS en médecine et santé, Paris, Les Belles Lettres, 2011.

Bonah, Christian, Visier Laurent, « De l'éducation sanitaire à la promotion de la santé », in Bonah C., Haxaire C., Mouillie J.-M., Penchaud A.-L., Visier L. (dir.), Sciences humaines, médecine et santé. Manuel du Collège des enseignants de SHS en médecine et santé, Paris, Les Belles Lettres, 2011, pp. 268-275.

Bonah, Christian ; Alexis Zimmer, «Le calvaire du Steinach Film (1922/23). Représentations du médecin dans le film de recherche et d'enseignement médical, Sociétés \& Représentations, 28, 2009, pp. 87-106.

Bonah, Christian ; Laukötter, Anja, "Moving pictures and medicine in the first half of the 20th century: Some notes on international historical developments and the potential of medical film research", Gesnerus, 66, 2009, pp. 121-145.

Bonah, Christian ; Lowy, Vincent, « D’Erbkrank (1934-36) à Opfer der Vergangenheit (1937) : les représentations du handicap mental dans le cinéma de propagande nazi », in Meyer, Christian, Normes et normalisation du travail, Annecy, GEPSO, 2010, pp. 35-49.

Boon, Timothy, Films and the Contestation of Public Health in Interwar Britain, London, PhD thesis, University of London, 1999.

Boon, Timothy, Films of Fact A history of Science in Documentary Films and Television, London, Wallflower, 2008.

Cantor, David, "Uncertain enthusiasm: The American Cancer Society, public education, and the problems of movies, 1921-1960," Bulletin of the History of Medicine, 81, 2007,p. 39-69.

Cartwright, Lisa, Screening the body. Tracing medicine visual culture, Minneapolis, 1995.

Chervin, Jacqueline, «Le traitement des thématiques scientifiques dans le journal télévisé depuis 1949. », in Leboeuf, Claude ; Pelissier, Nicolas (éds), « Communiquer l'information scientifique - éthique du journalisme », Paris,, L'Harmattan, 2003. 
Cheveigné, Suzanne de ; Chervin, Jacqueline, « Biotechnologies et médias », Communication \& Langages, 138, décembre, Paris, Armand Colin, pp. 43-56.

Contal, Maryse, Prevention pour la santé et médecine générale. Analyse et retour sur 29 films de prévention (1987-1999) contre le sida en France, thèse de médecine, Faculté de médecine de Strasbourg, 2013.

Corbin, Alain ; Courtine, Jean-Jacques ; Vigarello, Georges (éds.), Histoire du corps. Tome 3. Les mutations du regard. Le XXe siècle, Paris, Seuil, 2006.

Daston, Lorraine ; Peter Galison, Objectivity, New York : Zone Books 2007.

Debru, Claude (éd.), History of science and technology in education and training in Europe, Actes du congrès ALLEA (All European Academies), Strasbourg, 25-26 juin 1998. Luxembourg, Office for Official Publications of the European Communities, 1999.

Delage, Christian ; Guigueo, Vincent, L'historien et le film, Paris, Folio Histoire Gallimard, 2004.

Delage, Christian, La vision nazie de l'histoire à travers le cinéma documentaire du Troisième Reich, Lausanne, Éditions L'âge d'homme, 1989.

Ellenbrand, Petra, Die Volksbewegung und Volksaufklärung gegen Geschlechtskrankheiten in Kaiserreich und Weimarer Republik, Marburg, Görich \& Weiershäuser, 1999.

Elsaesser, Thomas, "Archives and Archaeologies. The Place of Non-Fiction Film in Contemporary Media," in Hediger, Vinzenz; Vonderau, Patrick (eds.), Films that Work. Industrial Film and the Productivity of Media, Amsterdam, Amsterdam University Press, 2009, pp. 19-34.

Fauque, Danielle «La « longue marche » d'un enseignement de l'histoire des sciences et des techniques, Tréma, 2006. http://trema.revues .org/83, mis en ligne le 01 octobre 2008.

Ferro, Marc, Cinéma et histoire, Paris, Folio Histoire Gallimard, 1977/1993.

Fleury, Béatrice (éd.), L'image empêchée - Du côté de la censure, Revue Champs Visuels, ${ }^{\circ} 11$, octobre 1998, Paris, L'Harmattan, 1998.

Flexner, Abraham, Medical Education in Europe. A report to the Carnegie Foundation for the Advancement of Teaching, Bull. n6, New York, 1912, pp. 119-122.

Fox, Daniel M. ; Lawrence, Christopher, Photographing medicine: images and power in Britain and America since 1840, New York, 1988.

Gaycken, Oliver, The Swarming of Life": Moving Images, Education, and Views through the Microscope, Science in Context, 24:3, 2011, pp 361-380.

Gaycken, Oliver, "Beauty of Chance": Film ist », Journal of Visual Culture, 11:3, 2012, pp. 307-327.

Gerverau, Laurent, Les images qui mentent, Histoire du visuel au XX $X^{\mathrm{e}}$ siècle, Paris, Éditions Seuil, 2000.

Gilman, Sander L., Health and illness: images of difference, London, 1995.

Guibbert, Pierre ; Oms, Marcel, L’histoire de France au cinéma, CinémAction Hors-série, 1993. London/ New York, Wallflower Press, 2005.

Jordanova, Ludmilla, « Medicine and Visual Culture », Social History of Medicine, 3, 1990, pp. 89-99.

Jung, Uli ; Loiperdinger, Martin (éds.), Geschichte des dokumentarischen Films in Deutschland. Band 1 Kaiserreich 1895-1918, Ditzingen, Reclam, 2005.

Keitz, Ursula von, Im Schatten des Gesetzes: Schwangerschaftskonflikt und Reproduktion im deutschsprachigen Film 1918 bis 1933, Marburg, Schüren, 2005.

Köhne, Julia Barbara, Kriegshysteriker. Strategische Bilder und mediale Techniken militärpsychiatrischen Wissens (1914-1920), Husum, Matthiesen, 2009.

Krause, Marcus, Pethes, Nicolas (éds.), Mr. Münsterberg und Dr. Hyde. Zur Filmgeschichte des Menschenexperiments, Bielefeld, Transcript, 2007.

Kreimeier, Klaus ; Ehmann, Antje ; Goergen, Jeanpaul (éds.), Geschichte des dokumentarischen Films in Deutschland. Band 2 (Weimarer Republik 1918-1933), Ditzingen, Reclam, 2005.

Kuhn, Annette, Cinéma, Censorship and Sexuality 1909-1925, London/NewYork, Routledge, 1988. 
Les sciences humaines dans les parcours scientifiques et techniques professionnalisants

Leblanc, Gérard, Quand l'entreprise fait son cinéma. La médiathèque de Rhône-Poulenc (1972-1981), Vincennes, 1983.

Lederer, Susan, "Dark Victory: Cancer and Popular Hollywood Film", Bulletin of the History of Medicine, 81, 2007, pp. 94-115.

Lefebvre, Thierry Cinéma et discours hygiéniste (1890-1930), Paris, Université Paris III, Thèse UFR Cinéma et audiovisuel, 1996.

Lefebvre, Thierry, La chair et le cellulö̈. Le cinéma chirurgical du docteur Doyen, Brionne, Jean Doyen Editeur, 2004.

Lepicard, E. et Fridman, K., "Medicine Culture \& Cinema : A Workshop in Medical Humanities for Clinical Years", Medical Education, 37, 2003, pp. 1039-1040.

Lepicard, Etienne «La culture médicale au miroir du cinéma. Un atelier de medical humanities pour les années cliniques » in Bonah, Christian ; Rasmussen, Anne ; (éds.), Actes du colloque : 10 ans d'enseignement de sciences humaines et sociales en médecine, Faculté de Médecine de Strasbourg, Strasbourg, 2005, pp. 85-87.

Lowy, Vincent, Cinéma et mondialisation. Une esthétique des inégalités, Paris, Le Bord de l'eau, coll. «Mondialisation, culture et com », 2011.

Lowy, Vincent, Guerre à la guerre ou le pacifisme dans le cinéma français (1936-1940), Paris, L'Harmattan, 2006.

Lowy, Vincent, L'histoire infilmable, les camps d'extermination nazis à l'écran, Paris, L'Harmattan, 2001.

Lowy, Vincent, Marcel Ophuls, Paris, Le Bord de l'eau, 2008.

Maehle, Andreas Hoger, "The Search for Objective Communication: Medical Photography in the Nineteenth Century”, in Renato G. Mazzolini, Non-Verbal Communication in Science Prior to 1900, Florence, 1993, pp. 563-586.

Martinet, Alexis, Le Cinéma et la science, Paris, CNRS Editions, 1994.

Matthes, Joachim, "The operation called "Vergleich"", in Matthes, Joachim, Zwischen den Kulturen? Die Sozialwissenschaften vor dem Problem des Kulturvergleichs, Soziale Welt Sonderband 8, Göttingen, 1992, pp. 75-99.

Neuman, Dominique, « Bobigny : 25 ans d'expérience en sciences humaines et sociales », in Bonah, Christian ; Rasmussen, Anne (éds.), Actes du colloque : 10 ans d'enseignement de sciences humaines et sociales en médecine, Faculté de Médecine de Strasbourg, Strasbourg, 2005, pp. 9-10.

Orgeron, Devin ; Orgeron, Marsha and Dan Streible (éds.), Learning with the Lights Off. Educational Film in the United States, New York, Oxford University Press, 2011.

Osolin, Janis, Rekonstruktion: Geschichte und Geschichten im Film, Basel, 1988.

Ostherr, K., "Invisible Invaders: The Global Body in Public Health Films", in Friedman L.D. (éd.), Cultural Sutures: Medicine, Media and Morals, Durham, Duke University Press, 2004, pp. 299-314.

Ostherr, Kirsten, Cinematic Prophylaxis: Globalization and Contagion in the Discourse of World Health, Durham, Duke University Press, 2005.

Parascandola, John, "VD at the movies: PHS Films of the 1930s and 1940s", Public Health Reports, 111, 2, 1996, pp. 173-175.

Pastre, Béatrice de ; Lefebvre, Thierry (éds.), Filmer la science, comprendre la vie : le cinéma de Jean Comandon, Paris, CNC, 2012.

Paulsen, Friedrich, Die deutschen Universitäten und das Universitätsstudium, Berlin, 1902. Paulsen, Friedrich, Geschichte des gelehrten Unterrichts, Berlin, 1921.

Pernick, Martin, “Thomas Edison's Tuberculosis Films: Mass Media and Health Propaganda", Hasting Center Report, 8, 1978, pp. 21-27.

Pernick, Martin, The black stork : eugenics and the death of "defective" babies in American medicine and motion pictures since 1915, Oxford University Press, New York, 1996.

Pessis, Georges, Entreprise et Cinéma. Cent ans d'images. Paris, La Documentation Française, 1997. 
Podoll, K., Lüning, J., "Geschichte des wissenschaftlichen Films in der Nervenheilkunde”, Fortschritte der Neurologie Psychiatrie, 3, 1998, pp. 122-132.

Rasmussen, Anne, «En revenir aux sources : l'usage des images dans la formation médicale», in Bonah, Christian ; Rasmussen, Anne (éds.), Actes du colloque : 10 ans d'enseignement de sciences humaines et sociales en médecine, Faculté de Médecine de Strasbourg, Strasbourg, 2005, pp. 90-93.

Reichert, Ramón, Im Kino der Humanwissenschaften. Sutdien zur Medialisierung des wissenschaftlichen Wissens, Bielefeld, Transcript, 2007.

Reim, Ulrike, "Der" Robert-Koch"-Film (1939) von Hans Steinhoff. Kunst oder Porpaganda?", in Benzenhöfer, Udo, Eckart, Woilfgang U. (éds.), Medizin im Spielfilm des Nationalsozialismus, Tecklenburg, Burgverlag, 1990, pp. 22-33.

Riehl-Halen, Heidrun, Der deutschsprachige Tuberkulosefilm in der medizinischen Aus-und Weiterbildung sowie in der Volksaufklärung, Heidelberg, Thesis University Heidelberg, 1998.

Ruebrecht, Caroline, Les films médico-scientifiques réalisés par Eric Duvivier dans les années 19601970 sur le thème de la psychiatrie, Mémoire de Master, Université de Strasbourg, 2012.

Sappol, Michael, "Visionary anatomies and the great divide: art, science and the changing conventions of anatomical representation, 1500-2003", in National Academy of Sciences (ed.), Visionary Anatomies, Washington, 2004, pp. 1-6, 37-41.

Schmidt, Ulf, "Der Blick auf den Körper. Sozialhygienische Filme, Sexualaufklärung und Propaganda in der Weimarer Republik", in Hagener, Michael (éd.), Geschlecht in Fesseln, Sexualität zwischen Aufklärung und Ausbeutung im Weimarer Kino 1918-1933, München 2002, pp. 23-46.

Schubring, Gert, "Einsamkeit und Freiheit" neu besichtigt. Universitätsreformen und Disziplinenbildung in Preussen als Modell für Wissenschaftspolitik im Europa des 19. Jahrhunderts, Stuttgart, Franz Steiner Verlag, 1991, pp. 276-326.

Self, D.J. et al., "Teaching Medical Ethics by Using Film Discussion”, Academic Medicine, 68, 1993, pp. 383-385.

Sorlin, Pierre, Sociologie du cinéma, Paris, Éditions Aubier Montaigne, 1977.

Stichweh, Rudolf, Etudes sur la genèse du système scientifique moderne, Presses universitaires de Lille, Lille, 1991.

Thévenard, Paul ; Tassel, G., Le cinéma scientifique Français, Paris, La Jeune Parque, 1948.

Turner, Roy Steven, "Towards a disciplinary order of sciences", in Minerva, 24, 1986, pp. 495-502.

Verdicchio, Dirk: Das Publikum des Lebens. Zur Soziologie des populären Wissenschaftsfilms. Bielefeld, Transcript, 2010.

Vignaux, Valérie, Jean-Benoît Lévy ou le corps comme utopie, Paris, Association Française de recherche sur le cinéma, 2007.

Vonderau, Patrick, "Vernacular Archiving. An interview with Rick Prelinger", in Hediger, Vinzenz; Vonderau, Patrick (éds.), Films that Work. Industrial Film and the Productivity of Media, Amsterdam, Amsterdam University Press, 2009, pp. 51-61.

Zimmermann, Peter ; Hoffmann, Kay (éds.), Geschichte des dokumentarischen Films in Deutschland. Band. 3 "Drittes Reich” 1933-1945, Ditzingen, Reclam, 2005.

Zimmermann, Yvonne, "What Hollywood is to America, the corporate film is to Switzerland". Remarks on industrial film as utility film", in Hediger, Vinzenz; Vonderau, Patrick (eds.), Films that Work, 2009, pp. 101-117.

Zimmermann, Yvonne, Schaufenster Schweiz. Dokumentarische Gebrauchsfilme 1896-1964, Zürich, Limmat Verlag 2011. 